bioRxiv preprint doi: https://doi.org/10.1101/2021.12.10.472093; this version posted December 11,2021 . The copyright holder for this preprint

(which was not certified by peer review) is the author/funder, who has granted bioRxiv a license to display the preprint in perpetuity. It is made available under aCC-BY-NC-ND 4.0 International license.

\title{
Chloride-dependent mechanisms of multimodal sensory discrimination and neuropathic sensitization in Drosophila
}

Nathaniel J. Himmel ${ }^{1}$, Akira Sakurai ${ }^{1}$, Jamin M. Letcher ${ }^{1}$, Atit A. Patel ${ }^{1}$, Shatabdi Bhattacharjee ${ }^{1}$, Maggie N. Benson ${ }^{1}$, Thomas R. Gray ${ }^{1}$, Gennady S. Cymbalyuk ${ }^{1}$, and Daniel N. Cox ${ }^{1, *}$

1 - Neuroscience Institute, Georgia State University, Atlanta, GA 30303, USA

* corresponding author (dcox18@gsu.edu) 


\section{Abstract}

Individual sensory neurons can be tuned to many stimuli, each driving unique, stimulus-relevant behaviors, and the ability of multimodal nociceptor neurons to discriminate between potentially harmful and innocuous stimuli is broadly important for organismal survival. Moreover, disruptions in the capacity to differentiate between noxious and innocuous stimuli can result in neuropathic pain. Drosophila larval Class III (CIII) neurons are peripheral noxious cold nociceptors and gentle touch mechanosensors; high levels of activation drive cold-evoked contraction (CT) behavior, while low levels of activation result in a suite of touch-associated behaviors. However, it is unknown what molecular factors underlie CIII multimodality. Here, we show that the TMEM16/anoctamins subdued and white walker (wwk; CG15270) are required for cold-evoked CT, but not for touch-associated behavior, indicating a conserved role for anoctamins in nociception. We also evidence that CIII neurons make use of atypical depolarizing chloride currents to encode cold, and that overexpression of ncc69-a fly homologue of NKCC1-results in phenotypes consistent with neuropathic sensitization, including behavioral hypersensitization and spontaneous nociceptor activity, making Drosophila CIII neurons a candidate system for future studies of the basic mechanisms underlying neuropathic pain. 


\section{INTRODUCTION}

Noxious stimuli are transduced by high-threshold sensory neurons referred to as nociceptors, and these sensory neurons often respond to more than one stimulus type - a property called sensory poly- or multimodality ${ }^{1-5}$. Although the ability to differentiate between sensory modalities is inarguably important for organismal survival, it remains relatively poorly understood what molecular mechanisms facilitate sensory multimodality within single neurons or neural subtypes; and these systems are of direct importance human health, as the inability to discriminate between noxious and innocuous stimuli is thought to underlie chronic neuropathic pain ${ }^{6-8}$.

Like other animals, Drosophila melanogaster can sense and respond to noxious stimuli. In larvae, nociception primarily begins in peripheral dendritic arborization neurons of the Class III (CIII) and Class IV (CIV) subtypes. CIV neurons are polymodal high temperature, mechanical, and chemical nociceptors, and activation of class IV neurons by any one of these sensory modalities elicits a corkscrew-like rolling behavior ${ }^{9-13}$. In contrast, CIII neurons are multimodal cold nociceptors and gentle-touch mechanosensors, and activation of CIII neurons drives stimulus-specific behaviors: acute noxious cold primarily elicits a highly stereotyped, bilateral contraction (CT) response, wherein the body rapidly shortens along the head-to-tail axis ${ }^{14}$, while gentle touches elicit a suite of behaviors, including head-withdrawal, head casting/turning behavior, and reverse locomotion ${ }^{15}$.

CIII neurons function via a high-low threshold detection system, whereby high levels of CIII activation (and strong $\mathrm{Ca}^{2+}$ transients) are associated with $\mathrm{CT}$, and low levels of activation (and relatively modest $\mathrm{Ca}^{2+}$ transients) with touch-behaviors. This is evidenced in $\mathrm{Ca}^{2+}$ imaging experiments and via optogenetics, where strong optogenetic activation of CIII neurons elicits CT and less strong activation primarily drives head withdrawals ${ }^{14}$. However, molecular factors underlying this high-low threshold filter have not been elucidated. Given the relatively 
constrained function and modality-specific behavioral outputs of CIII, this system constitutes a good target for elucidating mechanisms underlying multimodality in single neural classes.

Stimulus-evoked CIII calcium transients are thought to occur as a result of the activation of Transient Receptor Potential (TRP) channels, a superfamily variably selection cation channels which participate in nociception across a wide variety of species ${ }^{16}$. In Drosophila, the TRP genes $P k d 2, N o m p C$, and Trpm are required for cold nociception and gentle touch mechanosensation ${ }^{14}$. In vertebrates, $\mathrm{Ca}^{2+}$-dependent channels of a variety of subtypes (e.g. anoctamin/TMEM16 channels ${ }^{17}$ ) interact with TRP channels in nociceptive systems in order to drive appropriate levels of neural activation, making them a potential candidate mechanism underlying cold and touch discrimination. It has been previously shown that the Drosophila gene subdued encodes a Ca ${ }^{2+}$-activated $\mathrm{Cl}^{-}$channel $(\mathrm{CaCC})^{18,19}$, and that it is necessary for high temperature-evoked rolling ${ }^{20}$. However, it is unknown whether or not subdued might function to encode stimulus-specific sensory information in multimodal neurons; we therefore questioned whether anoctamins might function in CIII in a modality-specific fashion.

In the present study we tested the hypothesis that anoctamins expressed in CIII are selectively required for cold nociception. We have found that the anoctamins subdued and white walker (wwk; CG15270) - here shown to be orthologous to human ANO1/2 and ANO8, respectively-are required for cold nociception. Interestingly, anoctamins participate in an excitatory capacity, suggesting that CIII neurons make use of excitatory $\mathrm{Cl}^{-}$currents, and we provide additional evidence that CIII neurons likely use depolarizing $\mathrm{Cl}^{-}$currents to selectively encode cold. Further, we demonstrate that overexpression of ncc69 (a fly homologue of NKCC1) is sufficient for driving a neuropathic pain-like state in Drosophila larvae - a phenotype mechanistically analogous to human neuropathic pain following spinal cord injury. 


\section{MATERIALS AND METHODS}

\section{Fly Strains}

All Drosophila melanogaster stocks were maintained at $24^{\circ} \mathrm{C}$ under a $12: 12$ light cycle. Genetic crosses were raised at $29^{\circ} \mathrm{C}$ under a $12: 12$ light cycle in order to accelerate development. $3^{\text {rd }}$ instar larvae were used for all experiments. Publicly available transgenic strains were originally sourced from the Bloomington Drosophila Stock Center (B) and the Vienna Drosophila Resource Center (v) and included: T2A-GAL4 ${ }^{\text {wwk }}$ (B76649); UAS-Aurora (B76327); UAS-mCD8::GFP (B5130); wwk ${ }^{\text {MI03516 }}$ (B36976); Df(2L)b87e25 (wwk df, B3138); subdued $^{M 115535}$ (B61082); Df(3R)Exel6184 (subdued df, B7663); UAS-subdued RNAi (v32472 and v108953); UAS-wwk RNAi (B28650 and B62282); UAS-ncc69 RNAi (B28682); and UASkcc RNAi (B34584). UAS-kcc was provided by Dr. Mark Tanouye; UAS-ncc69 was provided by Dr. Don van Meyel; UAS-subdued and GAL4 $4^{\mathrm{c} 240}$ were provided by Dr. Changsoo Kim. GAL4 ${ }^{19-12}$ is available upon request.

CIII::tdTomato and UAS-wwk were developed for this study and are available upon request. For $G A L 4^{C I I I}:: t d T o m a t o$ we utilized Gateway cloning to combine the R83B04 (CIII) enhancer with CD4-tdTomato by LR reaction (Invitrogen ref: 12538-120). Transgenic flies carrying a $2^{\text {nd }}$ chromosomal insertion of CIIl:tdTomato (docking site: VK37) were generated by Genetivision. The R83B04 enhancer-containing entry vector was provided by the FlyLight Project. pDEST-HemmarR2-the vector for enhancer driven CD4-tdTomato expression-was provided by Dr. Chun Han. For UAS-wwk, full-length cDNA was synthesized (GenScript) and subcloned into pUAST-attB. Transgenic UAS-wwk flies carrying a $3^{\text {rd }}$ chromosomal insertion of UAS-wwk (docking site: VK20) were generated by Genetivision.

\section{Class III isolation and qRT-PCR}


Isolation of CIII neurons followed a previously described protocol ${ }^{21}$. In brief, 40-50 third instar larvae with mCD8::GFP-tagged CIII neurons were collected and washed in $\mathrm{ddH}_{2} \mathrm{O}$, dissected using microdissection scissors, and then dissociated in PBS using a glass dounce. CIII neurons were then isolated using superparamagnetic beads (Dynabeads MyOne Steptavidin T1, Invitrogen) that were conjugated to biotinylated anti-mCD8a antibody (eBioscience). RNA was isolated from these neurons using the RNeasy Mini Kit (Qiagen) and qRT-PCR analysis was performed in triplicate using pre-validated Qiagen QuantiTect Primer Assays for CG15270 (QT00936754) and subdued (QT00978131). Data are reported as $\mathrm{C}_{\mathrm{q}}$ value \pm standard deviation.

\section{Cold Nociception Assay}

$3^{\text {rd }}$ instar larvae were raised in and collected from standard fly food vials, gently washed in water, then acclimated to a room temperature, moistened, black-painted aluminum arena. Once locomotion resumed, the arena was transferred to a prechilled Peltier device (TE Technologies, CP0031) under the control of a thermoelectric temperature controller (TE Technologies, TC-48-20). Behavior was recorded from above by a Nikon D5200 DSLR camera, and the first 5 seconds of footage following contact between the arena and Peltier plate were used for behavioral analysis. Videos were converted to the avi format by Video to Video software (https://www.videotovideo.org/), and then further processed in the FIJI distribution of ImageJ. Virtual stack images of larvae were converted to grayscale, thresholded, and subsequently skeletonized into single pixel-wide lines representing larval length from tip to tip. Length measurements were normalized to the length at time 0 , and CT was counted if the larvae passed the CT threshold at any point during the analysis. The CT threshold was determined as in previous studies, as the average peak $w^{1118}$ decrease in length $+1.5 x$ the standard deviation; this resulted in a CT threshold (here called strong CT) of approximately 0.7 
(also presented as -0.3 change in normalized body length or $30 \%$ reduction in body length).

Data are reported as \% CT and the peak magnitude of decrease in larval length.

\section{Mechanosensation Assay}

$3^{\text {rd }}$ instar larvae were collected, gently washed, then acclimated to the same arena used in cold plate assays. Mechanosensitivity was scored similarly to previous reports ${ }^{14,15}$ : in brief, animals were brushed on an anterior segment by a single paintbrush bristle and behavior was observed through a Zeiss Stemi 305 microscope. Gentle touch behaviors include pausing/hesitating, head/anterior withdrawal (AKA hunching), head casting/turning, and reverse locomotion. Each subject was given 1 point if it performed one of these behaviors (for a maximum of 4 points per trial). Each animal was subject to three touch trials with 30 s intervals between each trial. The scores from the three trials were summed (for a maximum of 12 points per subject), and averaged across each genotype.

\section{Optogenetics}

$3^{\text {rd }}$ instar larvae were collected, gently washed, then acclimated to a glass plate. The plate was then transferred to a custom-built optogenetics-behavior rig fitted with bottommounted blue LED illumination and a top-mounted Canon Rebel T3i DSLR camera. Blue light, video recording, and larval tracking was handled automatically by Noldus EthoVision software; behavior was recorded for 5 seconds in the absence of blue light, for 10 seconds with blue light illumination, and for 5 additional seconds in the absence of blue light. Larval behavior was quantified by automated tracking of larval area from above.

\section{Microscopy}

For morphometric analyses, GFP tagged CIII neurons in $3^{\text {rd }}$ instar larvae were imaged on a Zeiss LSM 780 confocal microscope at 200X magnification. Images were collected as zstacks with a step size of $2.0 \mu \mathrm{m}$ and at $1024 \times 1024$ resolution. Maximum intensity projections of 
z-stacks were exported using Zen (blue edition) software and analyzed using the Analyze Skeleton ImageJ plugin. Metrics were compiled using custom Python algorithms freely available upon request.

To image GAL4 expression patterns, larvae were likewise imaged under Zeiss LSM 780 confocal microscope at various magnifications (scale bars present on relevant images), at various locations (indicated in relevant figures/legends). Images were viewed and exported from Zen (black edition) software and labeled in Adobe Illustrator.

\section{Electrophysiology}

Demuscled fillet preparations were made from $3^{\text {rd }}$ instar larvae were placed on the bottom of an experimental chamber $(200 \mu \mathrm{L})$ filled with HL-3 saline, which consisted of (in $\mathrm{mM}$ ) $70 \mathrm{NaCl}, 5 \mathrm{KCl}, 1.5 \mathrm{CaCl}_{2}, 20 \mathrm{MgCl}_{2}, 10 \mathrm{NaHCO}_{3}, 5$ trehalose, 115 sucrose, and $5 \mathrm{HEPES}(\mathrm{pH}$ 7.2). For the low-chloride saline, sodium chloride and magnesium chloride were replaced with sodium sulfate $\left(\mathrm{Na}_{2} \mathrm{SO}_{4}, 35 \mathrm{mM}\right)$ and magnesium sulfate $\left(\mathrm{MgSO}_{4}, 20 \mathrm{mM}\right)$. The osmolarity of the low-chloride saline was adjusted to become equal to HL-3 saline (355-360 mOsm) by adding sucrose (up to $170 \mathrm{mM}$ in total). The specimen was constantly perfused with HL-3 saline (30-40 $\mu \mathrm{L} / \mathrm{sec})$. Switching between HL-3 and low-chlorine saline was done by a three-way stopcock attached to the inlet tube.

Cold temperature stimulation was administered by passing the saline through an in-line solution cooler (SC-20, Warner Instruments, Hamden, CT, USA) connected to the controller device (CL-100, Warner Instruments, Hamden, CT, USA). The saline temperature was constantly monitored by a thermometer probe (BAT-12, Physitemp, Clifton, NJ, USA) placed adjacent to the preparation.

The spiking activity of a GFP-tagged CIII neurons was recorded extracellularly by using a borosilicate glass micropipette (tip diameter, 5-10 $\mu \mathrm{m}$ ) by applying gentle suction. The 
electrode was connected to the headstage of a patch-clamp amplifier (MultiClamp 700A, Molecular Devices, San Jose, CA, USA). All recordings were made from ddaA in the dorsal cluster of the peripheral sensory neurons. The amplifier's output was digitized at $10 \mathrm{kHz}$ sampling rate using a Micro1401 A/D converter (Cambridge Electronic Design, Cambridge, UK) and acquired into a laptop computer running Windows 10 (Microsoft, Redmont, WA, USA) with Spike2 software v. 8 (Cambridge Electronic Design, Cambridge, UK). Bursting spikes were identified as those with inter-spike intervals of less than 0.2 seconds.

\section{Phylogenetics}

Starting with previously characterized ANO sequences from human (NCBI CCDS), mouse (NCBI CCDS), and D. melanogaster (FlyBase), an ANO sequence database was assembled by Blastp against protein sequence/model databases at Ensembl and the Okinawa Institute of Science and Technology Marine Genomes Unit. In order to maximize useful phylogenetic information, only Blast hits $>300$ amino acids in length with an E-value less than 1E-20 were retained.

Next, CD-HIT was used to identify and remove duplicate sequences and predicted isoforms (threshold 90\% similarity), retaining the longest isoform to maximize phylogenetic information. Phobius was then used to predict TM topology; in order to further maximize phylogenetic information, sequences with fewer than five predicted TM segments were removed.

Sequences were thereafter aligned via MAFFT using default settings. The final phylogenetic tree was generated via IQ-Tree by the maximum likelihood approach, using an LG+R7 substitution model (as determined by ModelFinder). Branch support was calculated by ultrafast bootstrapping (UFboot, 2000 replicates). In order to identify duplication events, the maximum likelihood phylogeny was reconciled using NOTUNG 2.9.1. In order to formulate the 
most parsimonious interpretation of the resulting trees, weakly supported branches were rearranged (UFboot 95 cutoff) in a species-aware fashion against the species cladogram in

Figure 4A. Edge weight threshold was set to 1.0, and the costs of duplications and losses were set to 1.5 and 1.0, respectively. Trees were visualized using iTOL and Adobe Illustrator.

\section{Statistical Analyses}

Due to a growing call for statistical analyses that do not rely on $p$-values ${ }^{22-30}$, differences in mean and population proportions were analyzed using both traditional frequentist statistics and Bayesian alternatives.

Population proportions are presented as $\% \pm$ standard error of the proportion (SEP); differences in proportion were assessed by one-tailed (for $5^{\circ} \mathrm{C}$ experiments) or two-tailed (for $10^{\circ} \mathrm{C}$ experiments) z-test with a Bonferroni correction and the Bayesian A/B Test. All other measures are presented as mean \pm standard error of the mean (SEM) unless otherwise noted; differences were assessed by frequentist one- or two-way ANOVA with Dunnett's post-hoc tests and Bayesian equivalents ${ }^{31}$, or frequentist and Bayesian t-test (in the case of comparisons between only 2 groups). Electrophysiology low $\mathrm{Cl}^{-}$wash experiments were performed withinsubjects. For some subjects recordings were lost during the low $\mathrm{Cl}^{-}$or washout phase; to account for these missing data, differences were assessed using a linear mixed model and Bayesian repeated measures ANOVA (both analyzing the effect of different washes on firing frequency). z-tests were performed in Microsoft Excel, two-way ANOVA in GraphPad PRISM, and all other analyses in JASP (Bayesian analyses using default prior probability distributions or priors adjusted by the Westfall-Johnson-Utts method) ${ }^{32}$.

For frequentist analyses, statistical significance as assessed at $\alpha=0.05$. For Bayesian analyses, degree of support for rejecting the null hypothesis was inferred by computed Bayes Factors (BF), via a modification on the method originally proposed by Jeffreys ${ }^{33}$ : "null 
hypothesis supported" $\left(\mathrm{BF}_{10}<1\right)$; "weak" $\left(\mathrm{BF}_{10}\right.$ : 1-3); "substantial" $\left(\mathrm{BF}_{10}\right.$ : 3-10); "strong" $\left(\mathrm{BF}_{10}\right.$ : 10-30); "very strong" $\left(\mathrm{BF}_{10}: 30-100\right)$; "decisive" $\left(\mathrm{BF}_{10}>100\right)$.

All graphs were generated using GraphPad PRISM (GraphPad Software, La Jolla, California, USA) and Adobe Illustrator. Relevant $n$, adjusted $\mathrm{p}$, and $\mathrm{BF}_{10}$ values are listed in figure legends. In figures, asterisks $\left(^{*}\right)$ indicate statistical significance at $\alpha=0.05$; daggers ( $\dagger$ ) indicate weak evidence in favor of the alternative hypothesis; double daggers ( $¥)$ indicate at least substantial evidence in favor of the alternative hypothesis; and families of comparisons are grouped by overhead bars. 


\section{RESULTS}

\section{The anoctamins subdued and CG15270 are required for cold nociception}

Previous work has demonstrated that TRP channels are required for both CIIIdependent cold nociception and gentle touch mechanosensation ${ }^{14}$; however, no genes have been identified which are selectively required for CIII cold nociception. As vertebrate TRP channels function alongside anoctamin/TMEM16 channels in vertebrate nociceptors ${ }^{17}$, we first sought to identify anoctamins which might selectively participate in Drosophila cold nociception.

Cell-type specific transcriptomic data indicate that the anoctamin genes subdued and CG15270 are enriched in CIII cold nociceptors (Table S1; GEO accession GSE69353). In order to independently validate CIII expression of these genes, we performed qRT-PCR on isolated CIII neuron samples and found detectable expression of subdued $\left(C_{q}=24.53 \pm 0.12\right)$ and CG15270 $\left(\mathrm{C}_{\mathrm{q}}=28.29 \pm 0.09\right)$. To further validate these data, we drove expression of GFP using GAL4s under control of the native subdued and CG15270 promoters (Figure 1A), pairing these with a Class III::tdTomato fusion line. For subdued, we used the previously validated GAL4 ${ }^{c 240}$, which did in fact drive GFP expression in CIII nociceptors (Figure 1B). Consistent with previous reports $^{20}, G A L 4^{c 240}$ also drove GFP expression in CIV nociceptors (Figure S1). For CG15270, we made use of a CG15270-specific T2A-GAL4 (Trojan GAL4); this approach has been shown to be a strong indicator of gene expression ${ }^{34}$. CG15270 T2A-GAL4 drove expression of GFP in CIII nociceptors (Figure 1C). In fact, CG15270 appeared to be broadly expressed in larval peripheral sensory neurons (Figure S2A), as well as in the larval central nervous system (Figure S2B).

Using the previously developed cold plate assay ${ }^{35}$ we recorded larval behavior while delivering an acute ventral cold stimulus, which causes animals to contract $(C T)^{14}$ along the head-to-tail axis (Figure 1D). Larvae carrying homozygous Minos-mediated integration cassette 
alleles $^{36}$ (MiMIC, abbreviated as Mi) for subdued and CG15270 (Figure 1A) showed decreased cold sensitivity - fewer animals strongly CT in response to noxious cold (Figure 1E, Figure S3) and homozygous larvae had a reduced peak magnitude of CT (measured by maximum magnitude reduction in length normalized to initial length, Figure 1F). These data suggest that CG15270 and subdued are required for cold nociception. We therefore suggest the name white walker (wwk) for CG15270, as white walker mutant larvae are less sensitive to cold pain.

Larvae bearing a heterozygous subdued allele or a single chromosome with a deficiency covering the gene $(d f)$ displayed largely normal cold sensitivity (Bayesian analyses indicating evidence of a modest effect on \% strong CT in the heterozygous condition), while the mutant allele paired with the deficiency recapitulated the relatively strong homozygous phenotype, thereby providing evidence against the phenotype being the result of off-target mutations. Mutants carrying a heterozygous white walker allele behaved relatively normally, but a single deficiency resulted in reduced cold sensitivity. Further, the homozygous deficiency and the deficiency over MiMIC allele were lethal, meaning we could not rule out off-target effects by this approach alone.

\section{subdued and white walker are required in CIII neurons for CT behavior and cold-evoked}

\section{neural activity}

As subdued and white walker mutants had impaired cold nociception, we next sought to test the hypothesis that subdued and white walker function in CIII neurons (and further, to rule out off-target effects which could possibly be present in mutant backgrounds). To this end, we next knocked down the expression of subdued and white walker by UAS-mediated RNAi, targeting CIII neurons using the previously validated GAL4 $4^{19-12}$ driver $^{14}$. UP-TORR (https://www.flyrnai.org/up-torr/) was used to assess computationally predicted off-target effects for these RNAi constructs ${ }^{37}$; UP-TORR indicates that these constructs have no predicted offtarget effects. Consistent with the mutant phenotypes, CIII-specific knockdown of subdued or 
white walker resulted in reduced \% CT (Figure 2A, Figure S4) and reduced peak magnitude of CT (Figure 2B). Although subdued RNAi-2 showed a statistically insignificant decrease in \% strong CT, the phenotype is extremely similar to subdued RNAi-1 and Bayesian analyses indicate that there is substantial evidence in favor of the difference. For further experimentation we made use of the RNAi line with the strongest phenotype.

We further tested the hypothesis that subdued and white walker function in CIII neurons by assessing whether the mutant phenotypes were due to loss of function in CIII nociceptors, predicting that CIII driven UAS-white walker or UAS-subdued expression would rescue CT in mutant backgrounds. For these experiments, we used CIIl-targeting GAL4s located on chromosomes other than the chromosome containing the experimental gene (for white walker, GAL4 ${ }^{19-12}$; for subdued, GAL4 ${ }^{\text {nompC }}$ ). GAL4-UAS-mediated rescue in mutant backgrounds increased cold sensitivity (Figure 2A-B, Figure S5), indicating that subdued and white walker mutant phenotypes are due to selective anoctamin loss of function in cold nociceptors.

We next tested the hypothesis that anoctamins are involved in cold-evoked neural activity by making electrophysiological recordings in live, filet larvae during chilling. In control filet larvae, CIII firing frequency had an inverse relationship with temperature; chilling ramp resulted in bursting activity, while stable cold exposure resulted in tonic firing which increased in frequency with decreased temperature (Figure 2C, 2F). Clll-specific knockdown of subdued or white walker resulted in decreased cold-evoked CIII neural activity, particularly in steady-state tonic firing at a stable cold temperature (Figure 2C-E). Relative to controls, CIII-specific knockdown of subdued or white walker resulted in a reduction in overall firing frequency (spikes/sec) at both $15^{\circ} \mathrm{C}$ and $10^{\circ} \mathrm{C}$ (Figure 2F).

subdued and white walker are not required for mechanosensation and do not play a role on dendritogenesis 
We next tested the alternative hypothesis that these observed phenotypes reflect a loss of general excitability of CIII neurons. As CIII neurons are multimodal cold nociceptors and gentle touch mechanosensors, loss of general excitability or overall neural function would result in decreased gentle-touch mechanosensitivity; for example, previous reports have shown that loss of function of TRP channels required for cold nociception also results in defects in gentletouch mechanosensitivity ${ }^{14}$. Innocuous touch assays (based on the Kernan assay ${ }^{15}$ ) revealed that neither subdued or white walker loss of function affects gentle-touch mechanosensitivity

(Figure 3A-B). These results indicate that subdued and white walker are selectively required for CIII cold nociception.

We also tested the alternative hypothesis that decreased cold sensitivity was due to morphological defects in CIII dendritic arborization. CIII neurons labeled with GFP show no dendritic defects under subdued and white walker knockdown (Figure 3C-E); there were no quantitative differences in the number of dendritic branches, total dendritic length, or dendritic branch density (Figure 3F-H).

\section{The evolutionary history of anoctamins supports that subdued is part of the ANO1/ANO2 subfamily a $\mathrm{Ca}^{2+}$-activated $\mathrm{Cl}^{-}$channels}

While previous work has phylogenetically characterized anoctamins/TMEM16 channels ${ }^{38-40}$, their evolution and familial organization outside of vertebrates is relatively poorly understood, and therefore limits our ability to formulate hypotheses of function based on phylogeny. We therefore generated an anoctamin phylogeny which is inclusive of a wide range of animal taxa (Figure 4; Figures S6-S7). This phylogeny indicates that anoctamins are organized into 5 major metazoan subfamilies which predate the Cnidaria-Bilateria split (including a new subfamily we deem ANO11, which has not been previously described), and 6 major bilaterian subfamilies. subdued has been previously determined to encode a $\mathrm{Ca}^{2+}-$ activated $\mathrm{Cl}^{-}$channel $(\mathrm{CaCC}){ }^{19}$; consistent with these findings, this phylogeny evidences that 
subdued is a member of the bilaterian ANO1/2 subfamily of CaCCs. white walker is one of the more distantly related anoctamins and is of unknown function, but in contrast to subdued, has a single homologue in humans (both part of the metazoan ANO8 subfamily).

\section{CIII neurons make use of excitatory $\mathrm{Cl}^{-}$currents to encode noxious cold}

As subdued encodes an ANO1/ANO2 orthologue and $\mathrm{Ca}^{2+}$-activated $\mathrm{Cl}^{-}$channel ${ }^{19}$, and positively regulates cold nociception, we hypothesized that CIII neurons make use of atypical, excitatory $\mathrm{Cl}^{-}$currents to encode noxious cold.

Across animal taxa, neural $\mathrm{Cl}^{-}$homeostasis is maintained by differential expression of SLC12 co-transporters - in Drosophila, kazachoc (kcc) encodes an outwardly facing $\mathrm{K}^{+}-\mathrm{Cl}^{-}$ cotransporter ${ }^{41}$, while ncc69 encodes an inwardly facing $\mathrm{Na}^{+}-\mathrm{K}^{+}-\mathrm{Cl}^{-}$cotransporter ${ }^{42}$. The differential expression of these cotransporters can therefore modulate the membrane-potential effects of $\mathrm{Cl}^{-}$currents. Transcriptomic data indicates that $k c c$ is downregulated and $n c c 69$ is upregulated in CIII neurons (Table S1), indicating that CIII neurons may maintain relatively high intracellular $\mathrm{Cl}^{-}$concentrations, thereby facilitating depolarizing (and therefore excitatory) $\mathrm{Cl}^{-}$ currents. In order to manipulate $\mathrm{Cl}^{-}$homeostasis in CIII nociceptors we used RNAi and UAS constructs to knock down and overexpress ncc69 and kcc, and thereafter observed effects on noxious cold-evoked behavior.

Modulating the expression of SLC12 cotransporters affected cold-evoked behavior (Figure 5A-B, Figure S8). Knockdown of ncc69 and overexpression of $k c c$ - both of which would theoretically decrease intracellular $\mathrm{Cl}^{-}$concentration-resulted in reduced \% strong CT. This difference was statistically different for ncc69 RNAi, and there is substantial evidence according to Bayesian analyses for an effect in both ncc69 RNAi and kcc OE on \% strong CT. However, support for the $k c c O E$ phenotype was notably weaker, as there was no evidence for differences in average peak magnitude decrease in larval length. Further, knockdown of kcc and 
overexpression of ncc69-which would both theoretically increase intracellular $\mathrm{Cl}^{-}$ concentration-did not obviously affect sensitivity to $5^{\circ} \mathrm{C}$ noxious cold. Manipulating $\mathrm{Cl}^{-}$ physiology in this fashion did not affect gentle touch mechanosensitivity, indicating that $\mathrm{Cl}^{-}$ physiology is selectively required for CIII cold nociception (Figure S9)

These results are consistent with the hypothesis that $\mathrm{CIII} \mathrm{Cl}^{-}$currents are excitatory and selectively required for cold. However, these results are not alone sufficient to disprove the alternative hypothesis that $\mathrm{Cl}^{-}$currents are inhibitory; for example, inhibitory currents may be required for patterning neural activity in such a way to appropriately drive behavior, as is the case in CIV neurons with respect to hyperpolarizing $\mathrm{K}^{+}$currents ${ }^{43,44}$.

In order to further test these competing hypotheses, we drove CIII expression of Aurora, an engineered $\mathrm{Cl}^{-}$channel which gates in response to blue-light illumination ${ }^{45}$. Typically, Aurora is employed as an inhibitory optogenetic tool. If $\mathrm{CII} \mathrm{Cl}^{-}$currents are excitatory, one would expect optogenetic activation of these $\mathrm{Cl}^{-}$channels to result in CllI-mediated behaviors. In fact, blue-light illumination of freely locomoting $G A L 4^{19-12}>U A S$-Aurora larvae elicited CT behavior (frequently followed by head-casting and reverse locomotor behavior), indicating that $\mathrm{Cl}^{-}$ currents are excitatory and activate CIII neurons (Figure 5C-D). These findings collectively disprove the hypothesis that cold-evoked $\mathrm{CIII} \mathrm{Cl}^{-}$currents are inhibitory.

\section{Low extracellular $\mathrm{Cl}$ - concentrations and overexpression of ncc69 results in nociceptive sensitization}

We next modulated extracellular $\mathrm{Cl}^{-}$in our electrophysiology preparations in order to more directly observe the effect of the $\mathrm{Cl}^{-}$gradient on CIII activity. Decreasing extracellular $\mathrm{Cl}^{-}$ sensitized CIII neurons; bath application of low $\mathrm{Cl}^{-}$saline induced spontaneous CIII bursting and increased sensitivity to cooling (Figure 6), effects which could be washed out. Relative to 
controls, we observed increased spontaneous firing under low $\mathrm{Cl}^{-}$saline at room temperature, increased firing at $20^{\circ} \mathrm{C}$ and $15^{\circ} \mathrm{C}$, and no effect at $10^{\circ} \mathrm{C}$ (Figure 6B).

One of the hallmarks of neuropathic pain following spinal cord injury in mammals is upregulation of NKCC1 (a human orthologue of ncc69) in neurons involved in nociception, resulting in increased intracellular $\mathrm{Cl}^{-}$, and thereby aberrantly excitatory $\mathrm{Cl}^{-}$currents, leading to nociceptive sensitization and spontaneous nociceptor activity, much like we observed under low $\mathrm{Cl}^{-}$saline conditions ${ }^{46,47}$. In an attempt to genetically model neuropathic pain we overexpressed ncc69 in CIII neurons, predicting it would likewise cause nociceptive hypersensitivity.

Overexpression of ncc69 sensitized neurons to cooling and resulted in spontaneous, room temperature CIII activity (Figure 7A, 7C, 7D). Curiously, we did not observe deficits in CIII firing under ncc69 knockdown (Figure 7B).

We next assessed cold nociception in response to less-noxious cold $\left(10^{\circ} \mathrm{C}\right)$, which causes strong CT in only $\sim 30 \%$ of wild-type animals and is not affected by subdued or white walker loss of function (Figure 7E-F, Figure S10). Based on our electrophysiological observations, we predicted that overexpression of ncc69 and knockdown of kcc would result in cold hypersensitivity to less-noxious temperature. Consistent with these electrophysiological observations, GAL4-UAS-mediated overexpression of ncc69 resulted in increased cold sensitivity at less-noxious temperatures; more subjects strongly CT in response to less-noxious cold (Figure 7E, Figure S10) and the magnitude of CT was substantially stronger (Figure 7F). However, larvae were not sensitized to innocuous touch (Figure S9), and unexpectedly, the cold-sensitivity phenotype was not mirrored by kcc knockdown (a sensitization phenotype only barely more likely than no phenotype, as inferred by Bayesian analyses). 


\section{DISCUSSION}

Here, we have shown that CIII cold nociceptors make use of excitatory $\mathrm{Cl}^{-}$currents in order to selectively encode cold. Our current working hypothesis in light of these findings is that cold-evoked, TRP-channel mediated $\mathrm{Ca}^{2+}$ currents activate $\mathrm{Ca}^{2+}$-activated $\mathrm{Cl}^{-}$channels (CaCCs), which due to differential expression of ncc69 and $k c c$, results in depolarizing $\mathrm{Cl}^{-}$ currents, enhancing neural activation in response to cold (Figure 8). These results support a role for subdued, white walker, and ncc69 in selectively facilitating CIII-dependent cold nociception and not mechanosensation, thereby participating in mechanisms that allow CIII neurons to differentiate between sensory modalities.

As subdued has been previously characterized as a CaCC, its role is consistent with the hypothesis outlined above. However, the evolution of subdued has been implicitly debated in the literature ${ }^{18,19}$, with suggestion that it may be more closely related to ANO6 ${ }^{18}$. Our phylogenetic analysis strongly evidences that subdued is part of the bilaterian ANO1/ANO2 subfamily of CaCCs. Moreover, our phylogeny suggests that insects have no direct ANO6 homologue, as the diversification of ANO3, ANO4, ANO5, ANO6, and ANO9 occurred after the protostome-deuterostome split. The role of subdued in cold nociception therefore may constitute functional homology in the bilaterian ANO1/ANO2 subfamily, as mammalian ANO1 has been shown to participate in nociception alongside mammalian TRP channels ${ }^{48}$. However, the possibility of convergent evolution cannot yet be ruled due to the absence of evidence of function in other taxa.

In contrast, white walker has not been demonstrated to function as, or be closely related to, CaCCs. Our phylogeny evidences that white walker is part of the metazoan ANO8 subfamily; one important function of mammalian ANO8 is to tether the endoplasmic reticulum (ER) and plasma membrane $(\mathrm{PM})$, thereby facilitating inter-membrane $\mathrm{Ca}^{2+}$ signaling ${ }^{49}$. Therefore, a speculative hypothesis is that white walker likewise serves to couple the ER and PM, and that 
subsequently, ER-dependent $\mathrm{Ca}^{2+}$ signaling might promote the CIII cold response. However, ANO8 has been shown to conduct $\mathrm{Cl}^{-}$heterologously ${ }^{50}$, so $\mathrm{Cl}^{-}$channel function in Drosophila cannot be ruled out a priori. As white walker appears to be broadly expressed in neural tissues, white walker may function as a fundamental component of insect neural machinery, and is therefore likely to be a gene of interest in future studies.

In addition to the functions outlined above, anoctamins_-including subdued ${ }^{18}$ —are known to also function as lipid scramblases ${ }^{51}$. A plausible alternative hypothesis is therefore that subdued and/or white walker function as lipid scramblases as part of unidentified signaling cascades critical to noxious cold transduction.

The results of our ncc69 knockdown behavior, $\mathrm{Cl}^{-}$-channel optogenetics, and $\mathrm{Cl}^{-}$ electrophysiology experiments are consistent with the hypothesis that CIII neurons make use of atypical excitatory $\mathrm{Cl}^{-}$currents. However, we did not observe an effect on cold-evoked CIII activity in response to ncc69 knockdown (Figure 7B). As ncc69 knockdown behavioral defects are seen at $5^{\circ} \mathrm{C}$ (Figure 5A-B) and not at $10^{\circ} \mathrm{C}$ (Figure 7E-F), it may be the case that this knockdown only affects electrical activity at very noxious temperatures. Our inability to detect deficiencies in cold-evoked neural activity may therefore be due to limitations in our electrophysiology prep, which limit our ability to cool below $10^{\circ} \mathrm{C}$. These results are still curious, however, as subdued and white walker knockdowns result in electrophysiological defects at less-noxious $\left(10^{\circ} \mathrm{C}\right)$ and innocuous $\left(15^{\circ} \mathrm{C}\right)$ temperature drops (Figure 2D-F). Moreover, although we have substantial Bayesian evidence of an effect on \% strong CT under kcc overexpression (Figure 5A), this difference was not evidenced by traditional frequentist statistics, the phenotype did not clearly mimic ncc69 knockdown (Figure 5A), nor did we see a difference in the mean peak magnitude of CT response (Figure 5B). In totality, these results may suggest that $\mathrm{CIII} \mathrm{Cl}^{-}$homeostasis involves other cotransporters which can adapt in either function or expression in response to loss or gain of function. Given the importance of this 
system to behavior selection, $\mathrm{CIII} \mathrm{Cl}^{-}$homeostasis will make an interesting target for future experimentation.

Importantly, we have shown that overexpression of ncc69-a fly orthologue of NKCC1is sufficient for driving a neuropathic pain-like state in larvae. As misexpression of NKCC1 is a major factor associated with neuropathic pain in humans, we posit that altered larval cold nociception constitutes a new system in which to study neuropathic pain. Importantly, this system is wholly genetic, and does not require injury or other methods of invoking nociceptive hypersensitization, making it a high-throughput and easily accessible tool. Interestingly, RNAi knockdown of kcc did not mirror the ncc69 overexpression phenotype. We speculate that this is because native $k c c$ expression levels are low enough that knockdown does not sufficiently disrupt $\mathrm{Cl}^{-}$homeostasis. This might also be because of hypothetical unknown mechanisms of compensation, as discussed above.

While it has been often stated that neuropathic pain is maladaptive, there is growing support for the hypothesis that neuropathic pain has its mechanistic bases in adaptive nociceptive hypersensitization - a mechanism by which organisms are more readily able to respond to danger following insult ${ }^{48,52-63}$. Nerve injury has been previously shown to cause nociceptive hypersensitization in adult Drosophila, and has been hypothesized to be protective 57. Moreover, it has been recently shown that nociceptive hypersensitization of CIII neurons is coincident with cold acclimation, the mechanism by which insects adapt to dips in temperature ${ }^{64}$. One speculative hypothesis is that changes in expression levels of SLC12 transporters underlie these shifts in cold acclimation-induced cold sensitivity. This would be consistent with a study demonstrating that a number of genes in Drosophila involved in ion homeostasis are differentially regulated following cold acclimation ${ }^{65}$. If this speculation is veridical, insect thermal acclimation may serve as an example of how "maladaptive" injury and neuropathic sensitization can confer an adaptive advantage. It is therefore possible that these findings, and continued 
bioRxiv preprint doi: https://doi.org/10.1101/2021.12.10.472093; this version posted December 11, 2021. The copyright holder for this preprint (which was not certified by peer review) is the author/funder, who has granted bioRxiv a license to display the preprint in perpetuity. It is made available under aCC-BY-NC-ND 4.0 International license.

study, will lead to not only advances relevant to human health, but also better our understanding of nervous system evolution and the evolution of mechanisms underlying neuropathic sensitization and pain. 


\section{Acknowledgments}

This work is supported by NIH R01 NS115209-01 (to DNC and GSC). NJH was supported by NIH F31 NS117087-01, a GSU Brains \& Behavior Fellowship, and a Kenneth W. and

Georganne F. Honeycutt Fellowship. JML was supported by a GSU Brains \& Behavior

Fellowship. We thank Dr. Don van Meyel (McGill University), Dr. Mark Tanoye (UC Berkeley),

Dr. Changsoo Kim (Chonnam National University), Dr. Chun Han (Cornell University), and the FlyLight Project (Janelia Research Campus) for providing Drosophila stocks.

\section{Author Contributions}

Conceptualization, NJH and DNC; Methodology, NJH, AS, and DNC; Cold-plate assays, NJH, MNB, and TRG; Innocuous touch assays, NJH; Cell isolation at qRT-PCR, SB; Optogenetics, NJH and JML; Constructed custom optogenetics rig, AAP; Microscopy, NJH, JML, and AAP; Neural reconstructions and morphometric analyses, JML; Electrophysiology, AS; Phylogenetics, $\mathrm{NJH}$; Statistics and other formal analyses, NJH and AS; Characterized GAL4 ${ }^{\text {CIII }}$ and developed CIII::tdTomato fusion line, AAP; Developed UAS-wwk, NJH and SB; Writing - Original Draft, NJH; Writing - Review \& Editing - NJH, AS, JML, AAP, MNB, SB, TRG, GSC, and DNC;

Visualization, NJH, AS, JML, and GSC; Supervision, DNC; Funding acquisition, NJH, GSC, and DNC. 


\section{FIGURE LEGENDS}

Figure 1. subdued and white walker (CG15270) are expressed in CIII neurons and required for cold nociception. (A) Alleles, chromosomal deficiencies, and gene-specific GAL4s used in this study. (B-C) UAS-GFP driven under the control of GAL4 $4^{c 240}$ and T2A-GAL4 ${ }^{\text {wwk }}$ evidences that subdued and white walker are expressed in CIII neurons. (D) For cold plate assay, larvae were acclimated to a room temperature arena before being transferred to a pre-chilled cold plate. CT was identified by measuring the length of skeletonized larvae over the course of chilling. (E) \% of animals which strongly CT in response to noxious cold ( $\geq 30 \%$ reduction in body length). Mutations in subdued and white walker result in a reduced percent of larvae which strongly CT in response to noxious cold $\left(5^{\circ} \mathrm{C}\right) . w^{1118}(\mathrm{n}=30)$; subdued ${ }^{\mathrm{Mi} /+}\left(\mathrm{n}=30 ; \mathrm{p}=0.13 ; \mathrm{BF}_{10}=4.417\right)$; subdued $^{d f /+}\left(n=30 ; p=0.25 ; B_{10}=2.826\right) ;$ subdued $^{M i / M i}\left(n=30 ; p<0.001 ; B F_{10}=461.34\right) ;$ subdued $^{\text {Middf }}\left(\mathrm{n}=30 ; \mathrm{p}<0.001 ; \mathrm{BF}_{10}=997.24\right) ; w k^{\mathrm{Mi} /+}\left(\mathrm{n}=30 ; \mathrm{p}=0.45 ; \mathrm{BF}_{10}=2.00\right) ; w k^{d \mathrm{df}+}(\mathrm{n}=30$; $\left.\mathrm{p}<0.001 ; \mathrm{BF}_{10}=997.24\right) ; w w k^{\mathrm{Mi} / \mathrm{Mi}}\left(\mathrm{n}=30 ; \mathrm{p}<0.001 ; \mathrm{BF}_{10}=997.24\right)$. (F) Mean peak magnitude in larval contraction, corresponding to panel E. $w^{1118}(n=30)$; subdued ${ }^{M i /+}(n=30 ; p=0.58$; $\left.\mathrm{BF}_{10}=1.18\right) ;$ subdued $^{d \mathrm{f} / \mathrm{t}}\left(\mathrm{n}=30 ; \mathrm{p}=1 ; \mathrm{BF}_{10}=0.28\right) ;$ subdued $^{\mathrm{Mi} / \mathrm{Mi}}\left(\mathrm{n}=30 ; \mathrm{p}<0.001 ; \mathrm{BF}_{10}=228.63\right)$; subdued $^{\text {Mi/df }}\left(\mathrm{n}=30 ; \mathrm{p}=0.008 ; \mathrm{BF}_{10}=737.03\right) ; w k^{\mathrm{Mi} /+}\left(\mathrm{n}=30 ; \mathrm{p}=0.58 ; \mathrm{BF}_{10}=1.60\right) ; w k^{d \mathrm{df} /+}(\mathrm{n}=30$; $\left.\mathrm{p}=.003 ; \mathrm{BF}_{10}=324.11\right) ; w w k^{\mathrm{Mi} / \mathrm{Mi}}\left(\mathrm{n}=30 ; \mathrm{p}<0.001 ; \mathrm{BF}_{10}=433.18\right)$.

Figure 2. Anoctamins function in CIII neurons. (A) \% of animals which strongly CT in response to noxious cold ( $\geq 30 \%$ reduction in body length). CIII-specific knockdown ( $G A L 4^{19-12}$ ) of subdued and white walker result in a reduced percent of larvae which strongly CT in response to noxious cold. GAL4 control ( $\mathrm{n}=30)$; subdued RNAi-1 $\left(\mathrm{n}=30 ; \mathrm{p}=0.039 ; \mathrm{BF}_{10}=7.64\right) ;$ subdued RNAi-2 $\left(\mathrm{n}=27 ; \mathrm{p}=0.075 ; \mathrm{BF}_{10}=4.93\right) ;$ wwk RNAi-1 $\left(\mathrm{n}=30 ; \mathrm{p}=.002 ; \mathrm{BF}_{10}=71.17\right) ;$ wwk RNAi-2 $(\mathrm{n}=30 ;$ $\left.\mathrm{p}<0.001 ; \mathrm{BF}_{10}=138.15\right)$. GAL4-UAS-mediated CIII rescue of subdued and white walker in mutant backgrounds increased cold sensitivity. GAL4 $4^{\text {nompC }} ;$ subdued $^{M i M i}(\mathrm{n}=30) ;$ GAL4 ${ }^{\text {nompC }} / U A S-$ subdued;subdued $^{\text {MiMi }}\left(\mathrm{n}=30 ; \mathrm{p}=0.031 ; \mathrm{BF}_{10}=3.57\right) . w^{\text {Mi/Mi }} ; G A L 4^{19-12}(\mathrm{n}=30) ; w^{2} k^{M i / M i} ; G A L 4^{19-}$ 
${ }^{12} /$ AS - $w w k\left(n=30 ; \mathrm{p}<0.001 ; \mathrm{BF}_{10}=281.95\right)$. (B) Mean peak magnitude in larval contraction, corresponding to panel A. Knockdown: GAL4 control ( $n=30)$; subdued RNAi-1 $(n=30 ; p=0.049$; $\left.\mathrm{BF}_{10}=3.98\right) ;$ subdued RNAi-2 $\left(\mathrm{n}=27 ; \mathrm{p}=0.437 ; \mathrm{BF}_{10}=0.78\right) ;$ wwk RNAi-1 $(\mathrm{n}=30 ; \mathrm{p}=.005$; $\left.\mathrm{BF}_{10}=89.25\right) ; w w k R N A i-2\left(\mathrm{n}=30 ; \mathrm{p}<0.001 ; \mathrm{BF}_{10}=6932.18\right)$. Rescue: $G A L 4^{\text {nompC }} ;$ subdued ${ }^{\text {Mi/Mi }}$ (mutant, $\mathrm{n}=30$ ); GAL4 ${ }^{\text {nompC }} /$ UAS-subdued;subdued ${ }^{\text {Mi/Mi }}$ (CIII Rescue, $\mathrm{n}=30 ; \mathrm{p}=0.049 ; \mathrm{BF}_{10}=1.61$ ). $w_{w k}{ }^{M i / M i} ; G A L 4^{19-12}$ (mutant, $\left.\mathrm{n}=30\right) ; w k^{M i / M i} ; G A L 4^{19-12} /$ UAS-wwk (CIII rescue, $\mathrm{n}=30 ; \mathrm{p}<0.001$; $\left.\mathrm{BF}_{10}=83.59\right) .(\mathbf{C}, \mathbf{D}, \mathbf{E})$ Top: Heatmap representation of cold-evoked CIII activity $\left(10^{\circ} \mathrm{C}\right)$, with each line representing an individual sample prep. Middle: Representative traces of cold-evoked neural activity over graph of temperature ramp. Knockdown of subdued and white walker results in decreased cold-evoked firing, particularly in the steady-state tonic firing at steadystate temperature. Bottom: Representation of average frequency from population binned by 10 sec. Red and blue bars show the proportion of bursting vs tonic spiking activity. Knockdown of subdued and white walker results in visibly decreased tonic firing. (F) CIIl-specific knockdown of subdued and white walker results in decreased cold-evoked firing frequency at temperature ramps to $15^{\circ} \mathrm{C}$ and $10^{\circ} \mathrm{C}$. Room Temp (N=78): GAL4 control $(\mathrm{n}=32)$; white walker $R N A i(\mathrm{n}=24$; $\left.\mathrm{p}>0.99 ; \mathrm{BF}_{10}=0.274\right) ;$ subdued $R N A i\left(\mathrm{n}=22 ; \mathrm{p}=>0.99 ; \mathrm{BF}_{10}=0.279\right) .20^{\circ} \mathrm{C}(\mathrm{N}=65)$ : $\mathrm{GAL} 4$ control $(\mathrm{n}=25)$; white walker RNAi $\left(\mathrm{n}=22 ; \mathrm{p}=0.69 ; \mathrm{BF}_{10}=0.39\right)$; subdued $\operatorname{RNAi}(\mathrm{n}=18 ; \mathrm{p}=0.90$; $\left.\mathrm{BF}_{10}=0.32\right) .15^{\circ} \mathrm{C}(\mathrm{N}=68)$ : GAL4 control $(\mathrm{n}=27)$; white walker $R N A i(\mathrm{n}=22 ; \mathrm{p}=0.0082$; $\left.\mathrm{BF}_{10}=3.64\right)$; subdued $\operatorname{RNAi}\left(\mathrm{n}=19 ; \mathrm{p}<0.001 ; \mathrm{BF}_{10}=92.73\right) .10^{\circ} \mathrm{C}(\mathrm{N}=78)$ : GAL4 control $(\mathrm{n}=32)$; white walker RNAi ( $\left.\mathrm{n}=24 ; \mathrm{p}<0.001 ; \mathrm{BF}_{10}=3310.09\right)$; subdued $\operatorname{RNAi}(\mathrm{n}=22 ; \mathrm{p}<0.001$; $\left.\mathrm{BF}_{10}=44346.95\right)$.

Figure 3. subdued and white walker are not required for innocuous mechanosensation or CIII dendritogenesis. (A) There is no difference in gentle touch mechanosensation (sum Kernan touch scores, 3 trials for each sample) in either subdued and white walker mutants $(\mathrm{n}=10$ for each condition; $\left.p=0.20 ; B_{10}=0.62\right)$ or CIII-knockdown $(n=10$ for each condition; $p=0.70$; 
$\left.\mathrm{BF}_{10}=0.27\right)$. (B) For each genotype, there were no within-subjects effects in gentle touch sensation across trials. $w^{1118}\left(n=10 ; p=0.69 ; \mathrm{BF}_{10}=0.27\right) ; w w k^{M i / M i}\left(n=10 ; p=0.19 ; B F_{10}=0.85\right)$; subdued $^{\text {MiMi }}\left(\mathrm{n}=10 ; \mathrm{p}=0.42 ; \mathrm{BF}_{10}=0.42\right) ; \mathrm{GAL}_{4}$ control $\left(\mathrm{n}=10 ; \mathrm{p}=0.39 ; \mathrm{BF}_{10}=0.50\right) ;$ wwk RNAi $\left(\mathrm{n}=10 ; \mathrm{p}=0.37 ; \mathrm{BF}_{10}=0.47\right) ;$ subdued $R \operatorname{NAi}\left(\mathrm{n}=10 ; \mathrm{p}=0.80 ; \mathrm{BF}_{10}=0.25\right)$. (C-E) Representative neural traces of CIII ddaF neuron dendritic arbors under control and knockdown conditions. (F) Measures of total dendritic length show no differences between control and knockdown $(n=10$ for each condition; $\left.\mathrm{p}=0.48 ; \mathrm{BF}_{10}=0.34\right)$. $(\mathbf{G})$ There is no difference in the number of branches between control and knockdown ( $n=10$ for each condition; $\left.p=0.70 ; B F_{10}=0.27\right)$. (H) Dendritic branch density does not differ between control and knockdown ( $n=10$ for each condition; $\left.\mathrm{p}=0.81 ; \mathrm{BF}_{10}=0.24\right)$.

Figure 4. Anoctamin/TMEM16 channels form 5 metazoan and 6 nephrozoan clades. (A) Species, cladogram, and number of sequences used in this analysis. (B) Maximum likelihood phylogeny of animal anoctamins, rectified and rearranged against cladogram in A. subdued is a member of the ANO1/2 clade of nephrozoan calcium-activated chloride channels, and of the clade of metazoan anoctamins that includes mammalian ANO1/2/3/4/5/6/9. Mammalian ANO8 is homologous to white walker. Additionally, this phylogeny evidences a separate clade of ANOs of unknown function we have deemed ANO11 (cyan).

Figure 5. $\mathrm{CIII} \mathrm{Cl}{ }^{-}$currents are excitatory. (A) \% of animals which strongly $\mathrm{CT}$ in response to noxious cold ( $\geq 30 \%$ reduction in body length). CIII-specific knockdown (GAL4 ${ }^{19-12}$ ) of ncc69 results in a reduced percent of larvae which strongly CT in response to noxious cold. GAL4 control $(\mathrm{n}=30) ; \operatorname{ncc69} \operatorname{RNAi}\left(\mathrm{n}=29 ; \mathrm{p}=0.038 ; \mathrm{BF}_{10}=7.96\right) ; \operatorname{ncc69}$ OE $\left(\mathrm{n}=30 ; \mathrm{p}=1 ; \mathrm{BF}_{10}=0.58\right) ; k c c$ $\operatorname{RNAi}\left(\mathrm{n}=29 ; \mathrm{p}=0.91 ; \mathrm{BF}_{10}=0.99\right) ; \operatorname{kcc} O E\left(\mathrm{n}=30 ; \mathrm{p}=0.13 ; \mathrm{BF}_{10}=3.29\right)$. (B) Mean peak magnitude in larval contraction, corresponding to panel A. GAL4 control $(\mathrm{n}=30)$; ncc69 RNAi $(\mathrm{n}=29$; $\left.\mathrm{p}=0.025 ; \mathrm{BF}_{10}=46.38\right) ; n \operatorname{nc} 69 \operatorname{OE}\left(\mathrm{n}=30 ; \mathrm{p}=0.77 ; \mathrm{BF}_{10}=0.38\right) ; \operatorname{kcc} \operatorname{RNAi}(\mathrm{n}=29 ; \mathrm{p}=0.51$; $\left.\mathrm{BF}_{10}=0.68\right) ; \operatorname{kcc} O E\left(\mathrm{n}=30 ; \mathrm{p}=0.76 ; \mathrm{BF}_{10}=0.37\right)$. (C) Optogenetic activation of CIIl>Aurora 
activates CIII neurons, resulting in CT behavior. (D) CT behavior for control and CIIl>Aurora represented as larval area from above. Blue light activation of the $\mathrm{Cl}^{-}$channel Aurora causes a rapid reduction in normalized larval area from above, an indication of CT behavior. Comparison of minimum larval area measured during blue light illumination: GAL4 control ( $n=10) ; G A L 4^{19-12}$ $>$ UAS-Aurora $\left(\mathrm{n}=20 ; \mathrm{p}<0.001 ; \mathrm{BF}_{10}=134.78\right)$.

Figure 6. Modulating extracellular $\mathrm{Cl}^{-}$sensitizes CIII neurons. (A) Extracellular application of low $\mathrm{Cl}^{-}$saline to fileted electrophysiology preps causes spontaneous bursting activity. At room temperature, neurons are largely silent, but may show occasional, low frequency spiking (likely associated with mechanosensation from saline flow). (B) The presence of low $\mathrm{Cl}^{-}$saline causes spontaneous bursting and sensitizes CIII neurons to cooling. Room Temp: saline ( $n=12)$; low $\mathrm{Cl}^{-}$ $(n=12)$; wash $(n=8) ; p=0.003, B_{10}=1.89 .20^{\circ} \mathrm{C}$ : saline $(n=12)$; low $\mathrm{Cl}^{-}(n=12)$; wash $(n=8)$; $p=0.003, B F_{10}=66.38 .15^{\circ} \mathrm{C}$ : saline $(n=12)$; low $\mathrm{Cl}^{-}(n=12)$; wash $(n=7) ; p=0.011, \mathrm{BF}_{10}=14.51$. $10^{\circ} \mathrm{C}$ : saline $(n=12)$; low $\mathrm{Cl}^{-}(n=11)$; wash $(n=7) ; p=0.25, \mathrm{BF}_{10}=0.75$.

Figure 7. Overexpression of ncc69 causes nociceptive hypersensitization. (A, B, C) Top: Heatmap representation of cold-evoked CIII activity, with each line representing an individual sample prep. Middle: Representative traces of cold-evoked neural activity over graph of temperature ramp. Overexpression of ncc69 results in spontaneous nociceptor activity and increased cold sensitivity. Bottom: Representation of average spike frequency from population binned by $10 \mathrm{sec}$. Red and blue bars show the proportion of bursting vs tonic spiking activity. (D) Overexpression of ncc69 causes spontaneous neural activity and sensitizes neurons to cooling. Room Temp: GAL4 control ( $n=13) ; n c c 69$ RNAi $\left(n=12 ; p=0.99 ; B_{10}=0.22\right) ; U A S-n c c 69$ $\left(\mathrm{n}=12 ; \mathrm{p}<0.001 ; \mathrm{BF}_{10}=7.86\right) .20^{\circ} \mathrm{C}:$ GAL4 control $(\mathrm{n}=13) ;$ ncc69 RNAi $(\mathrm{n}=12 ; \mathrm{p}=0.96$; $\left.\mathrm{BF}_{10}=0.40\right) ; \operatorname{UAS}-n \operatorname{cc} 69\left(\mathrm{n}=11 ; \mathrm{p}=0.020 ; \mathrm{BF}_{10}=2.23\right) .15^{\circ} \mathrm{C}: \mathrm{GAL4}$ control $(\mathrm{n}=13) ;$ ncc69 RNAi $\left(n=12 ; p=0.90 ; B F_{10}=0.26\right) ; \operatorname{UAS}-n \operatorname{cc} 69\left(n=11 ; p=0.062 ; \mathrm{BF}_{10}=1.17\right) .10^{\circ} \mathrm{C}:$ GAL4 control $(n=13) ;$ ncc69 RNAi $\left(n=12 ; p=0.77 ; B_{10}=0.60\right) ; \operatorname{UAS}-n c c 69\left(n=11 ; p=0.0042 ; B_{10}=25.18\right) .(E) \%$ of 
animals which strongly CT in response to noxious cold $\left(10^{\circ} \mathrm{C}\right)$. CIII-specific (GAL4 $\left.4^{19-12}\right)$ overexpression of $n c c 69$ results in an increased percentage of strong CT in response to lessnoxious cold. $w^{1118}(n=28) ;$ GAL4 control $\left(n=30 ; p=1 ; B F_{10}=0.46\right) ; w w k^{M i / M i}(n=30 ; p=1$; $\left.\mathrm{BF}_{10}=0.69\right) ; w_{k} \operatorname{RNAi}\left(\mathrm{n}=30 ; \mathrm{p}=1 ; \mathrm{BF}_{10}=0.46\right) ;$ subdued $^{\mathrm{Mi} / \mathrm{Mi}}\left(\mathrm{n}=30 ; \mathrm{p}=1 ; \mathrm{BF}_{10}=0.69\right) ;$ subdued $\operatorname{RNAi}\left(\mathrm{n}=30 ; \mathrm{p}=1 ; \mathrm{BF}_{10}=0.46\right) ; \operatorname{UAS}-n \operatorname{cc} 69\left(\mathrm{n}=30 ; \mathrm{p}=0.026 ; \mathrm{BF}_{10}=28.79\right) ; \operatorname{kcc} \operatorname{RNAi}(\mathrm{n}=30 ; \mathrm{p}=1$; $\left.\mathrm{BF}_{10}=1.07\right)$. (F) Mean peak magnitude in larval contraction, corresponding to panel $\mathrm{E}$. $w^{1118}$ $(\mathrm{n}=28) ;$ GAL4 control $\left(\mathrm{n}=30 ; \mathrm{p}=1 ; \mathrm{BF}_{10}=0.27\right) ; w^{2} k^{\mathrm{Mi} / M i}\left(\mathrm{n}=30 ; \mathrm{p}=1 ; \mathrm{BF}_{10}=0.48\right) ;$ wwk RNAi $\left(\mathrm{n}=30 ; \mathrm{p}=1 ; \mathrm{BF}_{10}=0.52\right) ;$ subdued $^{\text {MiMi }}\left(\mathrm{n}=30 ; \mathrm{p}=1 ; \mathrm{BF}_{10}=0.27\right) ;$ subdued RNAi $(\mathrm{n}=30 ; \mathrm{p}=1$; $\left.\mathrm{BF}_{10}=0.27\right) ; \operatorname{UAS}-n c c 69\left(\mathrm{n}=30 ; \mathrm{p}=0.037 ; \mathrm{BF}_{10}=199.79\right) ; k c c \operatorname{RNAi}\left(\mathrm{n}=30 ; \mathrm{p}=1 ; \mathrm{BF}_{10}=0.29\right)$.

Figure 8. Graphical summary of hypothesis outlined in discussion: cold-evoked, TRP-channel mediated $\mathrm{Ca}^{2+}$ currents activate $\mathrm{Ca}^{2+}$-activated $\mathrm{Cl}^{-}$channels (CaCCs), which due to differential expression of $n c c 69$ and $k c c$, results in depolarizing $\mathrm{Cl}^{-}$currents, enhancing neural activation in response to cold. 


\section{REFERENCES}

1 Le Bars, D., Gozariu, M. \& Cadden, S. W. Animal Models of Nociception. Pharmacological Reviews 53, 597 (2001).

2 Himmel, N. J., Patel, A. A. \& Cox, D. N. Invertebrate Nociception. The Oxford Research Encyclopedia of Neuroscience, doi:10.1093/acrefore/9780190264086.013.166 (2017).

3 Sherrington, C. S. The Integrative Action of the Nervous System. (Yale University Press, 1906).

4 Smith, E. S. J. \& Lewin, G. R. Nociceptors: a phylogenetic view. Journal of comparative physiology. A, Neuroethology, sensory, neural, and behavioral physiology 195, 1089-1106, doi:10.1007/s00359-009-0482-z (2009).

5 Sneddon, L. U. Evolution of nociception in vertebrates: comparative analysis of lower vertebrates. Brain Research Reviews 46, 123-130, doi:https://doi.org/10.1016/j.brainresrev.2004.07.007 (2004).

6 Basbaum, A. I., Bautista, D. M., Scherrer, G. \& Julius, D. Cellular and molecular mechanisms of pain. Cell 139, 267-284, doi:10.1016/j.cell.2009.09.028 (2009).

7 Lumpkin, E. A. \& Caterina, M. J. Mechanisms of sensory transduction in the skin. Nature 445, 858, doi:10.1038/nature05662 (2007).

8 Woolf, C. J. \& Ma, Q. Nociceptors-Noxious Stimulus Detectors. Neuron 55, 353-364, doi:https://doi.org/10.1016/j.neuron.2007.07.016 (2007).

9 Himmel, N. J. et al. Drosophila menthol sensitivity and the Precambrian origins of transient receptor potential-dependent chemosensation. Philosophical Transactions of the Royal Society B: Biological Sciences 374, 20190369, doi:10.1098/rstb.2019.0369 (2019).

10 Hwang, R. Y. et al. Nociceptive neurons protect Drosophila larvae from parasitoid wasps. Current biology 17, 2105-2116, doi:10.1016/j.cub.2007.11.029 (2007).

11 Lopez-Bellido, R., Himmel, N. J., Gutstein, H. B., Cox, D. N. \& Galko, M. J. An assay for chemical nociception in Drosophila larvae. Philosophical Transactions of the Royal Society B: Biological Sciences 374, 20190282, doi:10.1098/rstb.2019.0282 (2019).

12 Neely, G. G. et al. TrpA1 Regulates Thermal Nociception in Drosophila. PLOS ONE 6, e24343, doi:10.1371/journal.pone.0024343 (2011).

13 Tracey, W. D., Wilson, R. I., Laurent, G. \& Benzer, S. painless, a Drosophila Gene Essential for Nociception. Cell 113, 261-273, doi:https://doi.org/10.1016/S0092-8674(03)00272-1 (2003).

14 Turner, H. N. et al. The TRP Channels Pkd2, NompC, and Trpm Act in Cold-Sensing Neurons to Mediate Unique Aversive Behaviors to Noxious Cold in Drosophila. Current Biology 26, 31163128, doi:10.1016/j.cub.2016.09.038 (2016).

15 Kernan, M., Cowan, D. \& Zuker, C. Genetic dissection of mechanosensory transduction: Mechanoreception-defective mutations of Drosophila. Neuron 12, 1195-1206, doi:https://doi.org/10.1016/0896-6273(94)90437-5 (1994).

16 Himmel, N. J. \& Cox, D. N. Transient receptor potential channels: current perspectives on evolution, structure, function and nomenclature. Proceedings of the Royal Society B: Biological Sciences 287, 20201309, doi:10.1098/rspb.2020.1309 (2020).

17 Takayama, Y., Uta, D., Furue, H. \& Tominaga, M. Pain-enhancing mechanism through interaction between TRPV1 and anoctamin 1 in sensory neurons. Proceedings of the National Academy of Sciences 112, 5213-5218, doi:10.1073/pnas.1421507112 (2015).

18 Le, T., Le, S. C. \& Yang, H. Drosophila Subdued is a moonlighting transmembrane protein 16 (TMEM16) that transports ions and phospholipids. J Biol Chem 294, 4529-4537, doi:10.1074/jbc.AC118.006530 (2019). 
Wong, X. M., Younger, S., Peters, C. J., Jan, Y. N. \& Jan, L. Y. Subdued, a TMEM16 family Ca ${ }^{2+}-$ activated $\mathrm{Cl}^{-}$channel in Drosophila melanogaster with an unexpected role in host defense. elife 2, e00862, doi:10.7554/eLife.00862 (2013).

20 Jang, W. et al. The anoctamin family channel subdued mediates thermal nociception in Drosophila. J Biol Chem 290, 2521-2528, doi:10.1074/jbc.M114.592758 (2015).

21 Iyer, E. P. R., Iyer, S. C., Sulkowski, M. J. \& Cox, D. N. Isolation and Purification of Drosophila Peripheral Neurons by Magnetic Bead Sorting. JoVE, e1599, doi:doi:10.3791/1599 (2009).

22 loannidis, J. P. A. What Have We (Not) Learnt from Millions of Scientific Papers with P Values? The American Statistician 73, 20-25, doi:10.1080/00031305.2018.1447512 (2019).

23 Kelter, R. Analysis of Bayesian posterior significance and effect size indices for the two-sample ttest to support reproducible medical research. BMC Medical Research Methodology 20, 88, doi:10.1186/s12874-020-00968-2 (2020).

24 Keysers, C., Gazzola, V. \& Wagenmakers, E.-J. Using Bayes factor hypothesis testing in neuroscience to establish evidence of absence. Nature Neuroscience 23, 788-799, doi:10.1038/s41593-020-0660-4 (2020).

25 Matthews, R., Wasserstein, R. \& Spiegelhalter, D. The ASA's p-value statement, one year on. Significance 14, 38-41, doi:https://doi.org/10.1111/j.1740-9713.2017.01021.x (2017).

26 Nuijten, M. B., Hartgerink, C. H. J., van Assen, M. A. L. M., Epskamp, S. \& Wicherts, J. M. The prevalence of statistical reporting errors in psychology (1985-2013). Behavior Research Methods 48, 1205-1226, doi:10.3758/s13428-015-0664-2 (2016). van Doorn, J. et al. The JASP guidelines for conducting and reporting a Bayesian analysis. Psychonomic Bulletin \& Review, doi:10.3758/s13423-020-01798-5 (2020).

28 Wasserstein, R. L. \& Lazar, N. A. The ASA Statement on p-Values: Context, Process, and Purpose. The American Statistician 70, 129-133, doi:10.1080/00031305.2016.1154108 (2016).

29 Wasserstein, R. L., Schirm, A. L. \& Lazar, N. A. Moving to a World Beyond "p[?] [0.05". The American Statistician 73, 1-19, doi:10.1080/00031305.2019.1583913 (2019).

30 Wetzels, R. et al. Statistical Evidence in Experimental Psychology: An Empirical Comparison Using 855 t Tests. Perspectives on Psychological Science 6, 291-298, doi:10.1177/1745691611406923 (2011).

31 Westfall, P. H., Johnson, W. O. \& Utts, J. M. A Bayesian perspective on the Bonferroni adjustment. Biometrika 84, 419-427, doi:10.1093/biomet/84.2.419 (1997). JASP (Version 0.14)[Computer Software] (2020). Jeffreys, H. The Theory of Probability. (Oxford University Press, 1961). Lee, P.-T. et al. A gene-specific T2A-GAL4 library for Drosophila. elife 7, e35574, doi:10.7554/eLife.35574 (2018).

35 Patel, A. A. \& Cox, D. N. Behavioral and Functional Assays for Investigating Mechanisms of Noxious Cold Detection and Multimodal Sensory Processing in Drosophila Larvae. Bio Protoc 7 , e2388, doi:10.21769/BioProtoc.2388 (2017).

36 Venken, K. J. T. et al. MiMIC: a highly versatile transposon insertion resource for engineering Drosophila melanogaster genes. Nat Methods 8, 737-743, doi:10.1038/nmeth.1662 (2011).

$37 \mathrm{Hu}, \mathrm{Y}$. et al. UP-TORR: online tool for accurate and Up-to-Date annotation of RNAi Reagents. Genetics 195 (2013).

38 Medrano-Soto, A. et al. Bioinformatic characterization of the Anoctamin Superfamily of Ca2+activated ion channels and lipid scramblases. PLOS ONE 13, e0192851, doi:10.1371/journal.pone.0192851 (2018).

39 Milenkovic, V. M., Brockmann, M., Stöhr, H., Weber, B. H. \& Strauss, O. Evolution and functional divergence of the anoctamin family of membrane proteins. BMC evolutionary biology 10, 319319, doi:10.1186/1471-2148-10-319 (2010). 
Wang, Y. et al. Phylogenetic, expression, and functional analyses of anoctamin homologs in Caenorhabditis elegans. American journal of physiology. Regulatory, integrative and comparative physiology 305, R1376-1389, doi:10.1152/ajpregu.00303.2012 (2013).

41 Hekmat-Scafe, D. S., Lundy, M. Y., Ranga, R. \& Tanouye, M. A. Mutations in the $\mathrm{K}^{+} / \mathrm{Cl}^{-}$ Cotransporter Gene kazachoc $(k c c)$ Increase Seizure Susceptibility in Drosophila. The Journal of Neuroscience 26, 8943-8954, doi:10.1523/jneurosci.4998-05.2006 (2006).

42 Leiserson, W. M., Forbush, B. \& Keshishian, H. Drosophila glia use a conserved cotransporter mechanism to regulate extracellular volume. Glia 59, 320-332, doi:https://doi.org/10.1002/glia.21103 (2011).

43 Onodera, K., Baba, S., Murakami, A., Uemura, T. \& Usui, T. Small conductance Ca ${ }^{2+}$-activated K ${ }^{+}$ channels induce the firing pause periods during the activation of Drosophila nociceptive neurons. elife 6, e29754, doi:10.7554/eLife.29754 (2017).

44 Terada, S.-I. et al. Neuronal processing of noxious thermal stimuli mediated by dendritic $\mathrm{Ca}^{2+}$ influx in Drosophila somatosensory neurons. elife 5, e12959, doi:10.7554/eLife.12959 (2016).

45 Wietek, J. et al. Anion-conducting channelrhodopsins with tuned spectra and modified kinetics engineered for optogenetic manipulation of behavior. Scientific Reports 7, 14957, doi:10.1038/s41598-017-14330-y (2017).

46 Hasbargen, T. et al. Role of NKCC1 and KCC2 in the development of chronic neuropathic pain following spinal cord injury. Annals of the New York Academy of Sciences 1198, 168-172, doi:https://doi.org/10.1111/j.1749-6632.2010.05462.x (2010).

47 Wilke, B. U., Kummer, K. K., Leitner, M. G. \& Kress, M. Chloride - The Underrated Ion in Nociceptors. Front Neurosci 14, 287-287, doi:10.3389/fnins.2020.00287 (2020).

48 Takayama, Y., Derouiche, S., Maruyama, K. \& Tominaga, M. Emerging Perspectives on Pain Management by Modulation of TRP Channels and ANO1. Int J Mol Sci 20, 3411, doi:10.3390/ijms20143411 (2019).

49 Jha, A., Chung, W. Y., Vachel, L. \& Maleth, J. Anoctamin 8 tethers endoplasmic reticulum and plasma membrane for assembly of $\mathrm{Ca}^{2+}$ signaling complexes at the ER/PM compartment. 38, doi:10.15252/embj.2018101452 (2019).

50 Tian, Y., Schreiber, R. \& Kunzelmann, K. Anoctamins are a family of $\mathrm{Ca}^{2+}$-activated $\mathrm{Cl}^{-}$channels. Journal of Cell Science 125, 4991-4998, doi:10.1242/jcs.109553 (2012).

51 Pedemonte, N. \& Galietta, L. J. V. Structure and Function of TMEM16 Proteins (Anoctamins). Physiological Reviews 94, 419-459, doi:10.1152/physrev.00039.2011 (2014).

52 Crook, R. J., Dickson, K., Hanlon, R. T. \& Walters, E. T. Nociceptive sensitization reduces predation risk. Curr Biol 24, 1121-1125, doi:10.1016/j.cub.2014.03.043 (2014).

53 Descalzi, G. et al. Neuropathic pain promotes adaptive changes in gene expression in brain networks involved in stress and depression. Sci Signal 10, eaaj1549, doi:10.1126/scisignal.aaj1549 (2017).

54 Gasull, X., Liao, X., Dulin, M. F., Phelps, C. \& Walters, E. T. Evidence that long-term hyperexcitability of the sensory neuron soma induced by nerve injury in Aplysia is adaptive. Journal of neurophysiology 94, 2218-2230, doi:10.1152/jn.00169.2005 (2005).

55 Géranton, S. M. Does epigenetic 'memory' of early-life stress predispose to chronic pain in later life? A potential role for the stress regulator FKBP5. Philosophical Transactions of the Royal Society B: Biological Sciences 374, 20190283, doi:10.1098/rstb.2019.0283 (2019).

56 Howard, R. B., Lopes, L. N., Lardie, C. R., Perez, P. P. \& Crook, R. J. Early-life injury produces lifelong neural hyperexcitability, cognitive deficit and altered defensive behaviour in the squid Euprymna scolopes. Philosophical Transactions of the Royal Society B: Biological Sciences 374, 20190281, doi:10.1098/rstb.2019.0281 (2019). 
$57 \quad$ Khuong, T. M. et al. Nerve injury drives a heightened state of vigilance and neuropathic sensitization in Drosophila. Science Advances 5, eaaw4099, doi:10.1126/sciadv.aaw4099 (2019).

58 Nesse, R. M. \& Schulkin, J. An evolutionary medicine perspective on pain and its disorders. Philosophical Transactions of the Royal Society B: Biological Sciences 374, 20190288, doi:10.1098/rstb.2019.0288 (2019).

59 Perrot-Minnot, M.-J., Banchetry, L. \& Cézilly, F. Anxiety-like behaviour increases safety from fish predation in an amphipod crustacea. Royal Society Open Science 4, 171558, doi:10.1098/rsos.171558 (2017).

60 Price, T. J. \& Dussor, G. Evolution: the advantage of 'maladaptive' pain plasticity. Current biology 24, R384-R386, doi:10.1016/j.cub.2014.04.011 (2014).

61 Walters, E. T. Adaptive mechanisms driving maladaptive pain: how chronic ongoing activity in primary nociceptors can enhance evolutionary fitness after severe injury. Philosophical Transactions of the Royal Society B: Biological Sciences 374, 20190277, doi:10.1098/rstb.2019.0277 (2019).

62 Walters, E. T. \& Williams, A. C. d. C. Evolution of mechanisms and behaviour important for pain. Philosophical Transactions of the Royal Society B: Biological Sciences 374, 20190275, doi:10.1098/rstb.2019.0275 (2019).

63 Williams, A. C. d. C. Persistence of pain in humans and other mammals. Philosophical Transactions of the Royal Society B: Biological Sciences 374, 20190276, doi:10.1098/rstb.2019.0276 (2019).

64 Himmel, N. J. et al. Identification of a neural basis for cold acclimation in Drosophila larvae. iScience, 102657, doi:https://doi.org/10.1016/j.isci.2021.102657 (2021).

65 MacMillan, H. A. et al. Cold acclimation wholly reorganizes the Drosophila melanogaster transcriptome and metabolome. Scientific Reports 6, 28999, doi:10.1038/srep28999 (2016). 
subdued and CG15270 (white walker) are expressed in CIII cold nociceptors

A subdued $|3 R| 2781$ bp

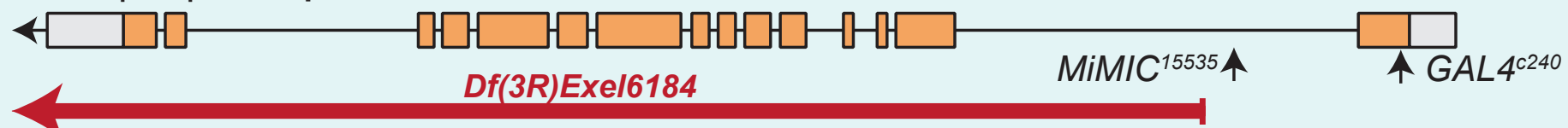

CG15270 | 2L | 3780 bp

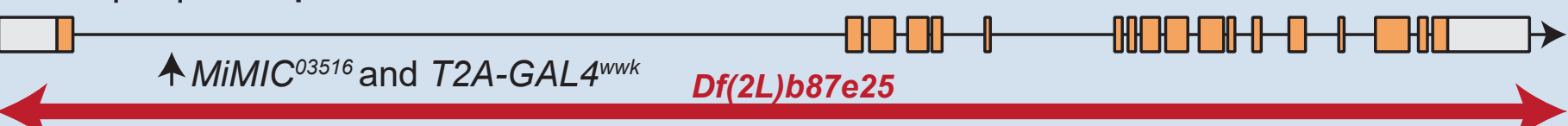

B

subdued $>$ GFP

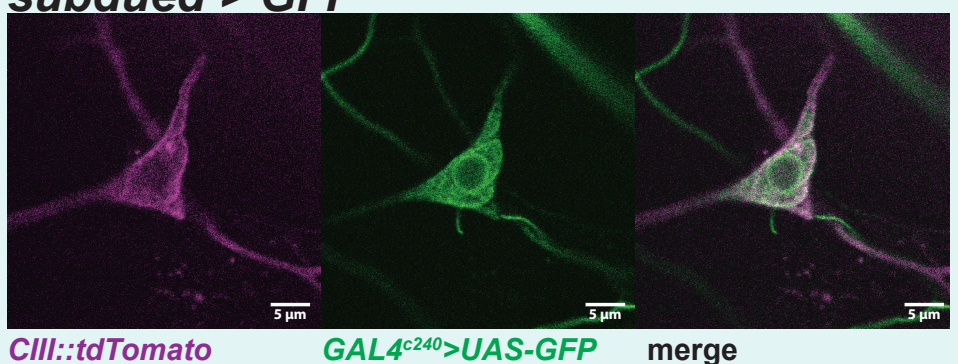

C

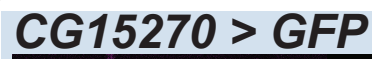

\section{subdued and white walker are required for cold nociception}

D

1 Acclimate to room temperature arena

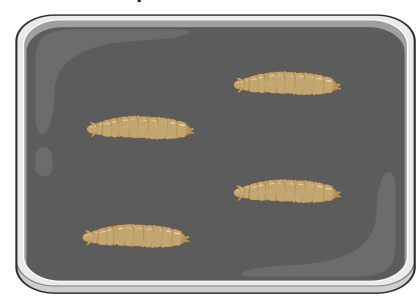

E
2 Move to prechilled Peltier device

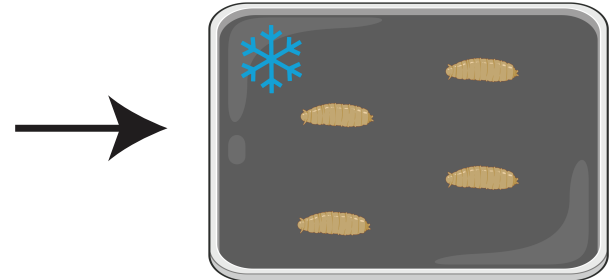

$\mathbf{F}$

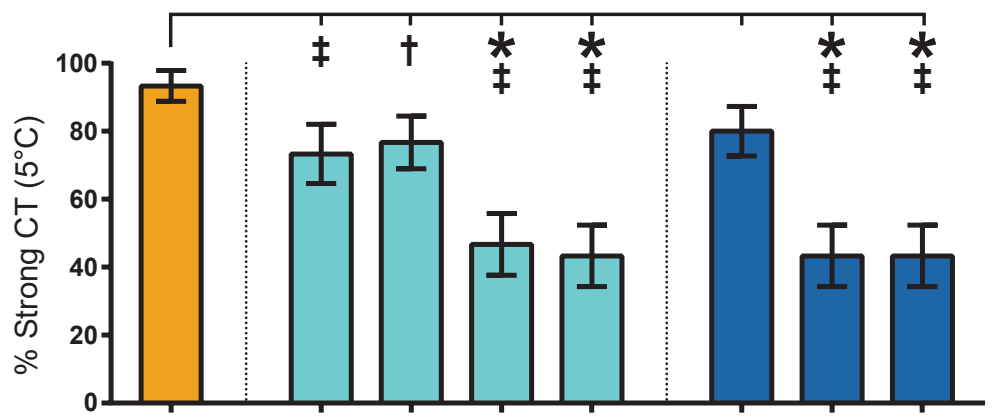
$\hat{N}^{1^{18}}$

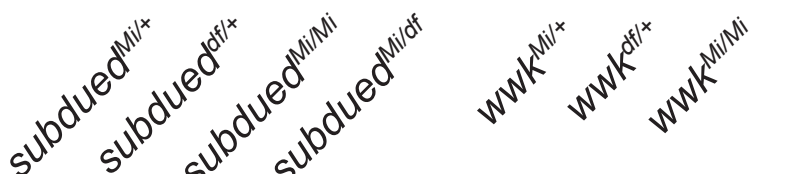

3 Skeletonize recodings of larval behavior and measure pixel length over $5 \mathrm{sec}$

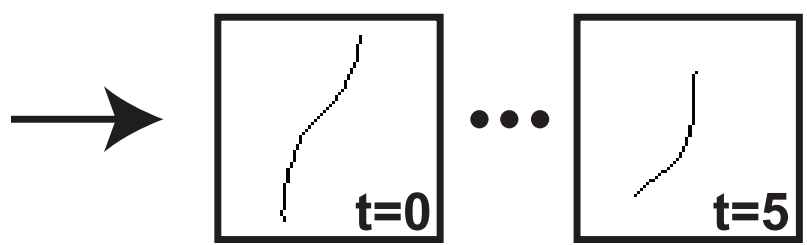

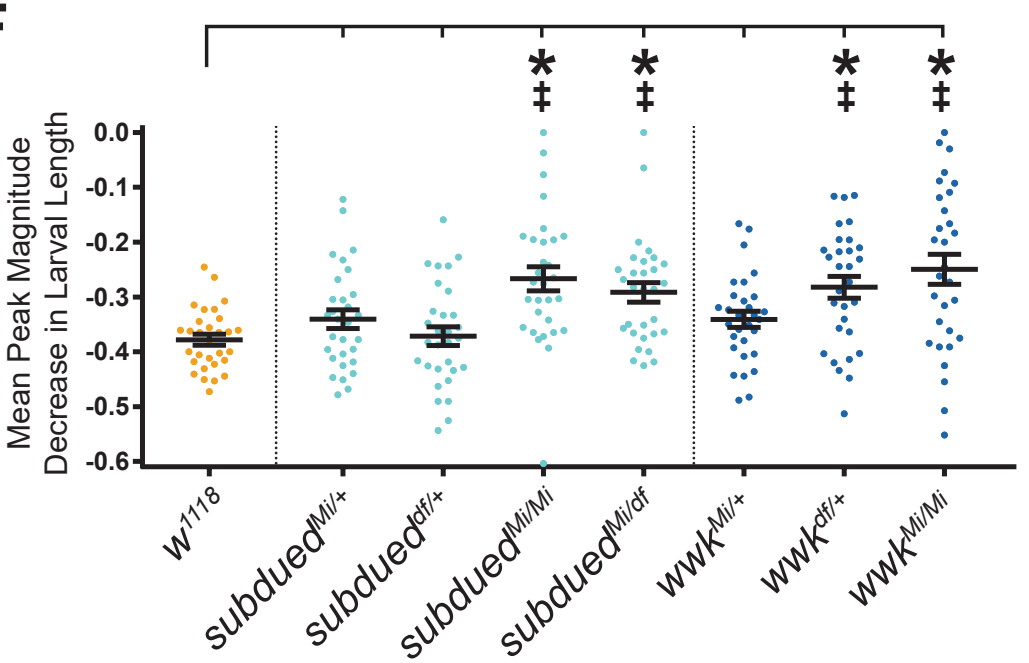




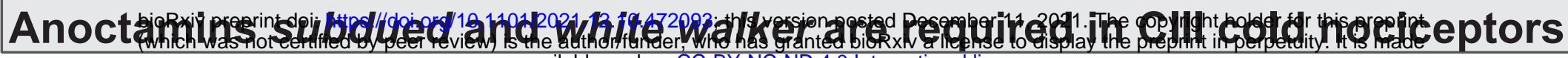

A RNAi knockdown
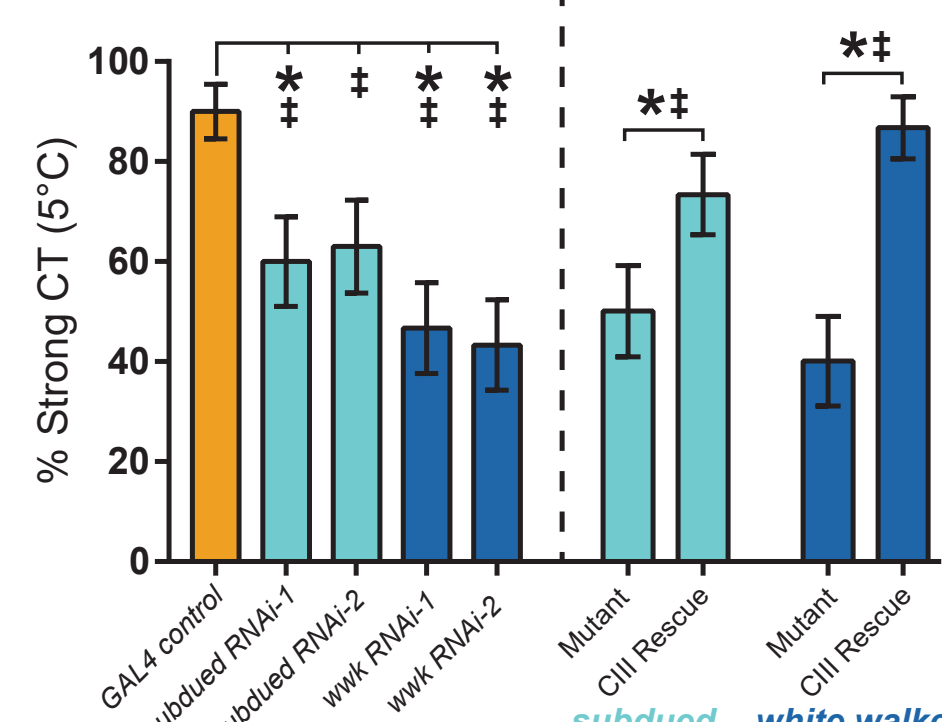

UAS rescue

subdued white walker
RNAi knockdown '

UAS rescue

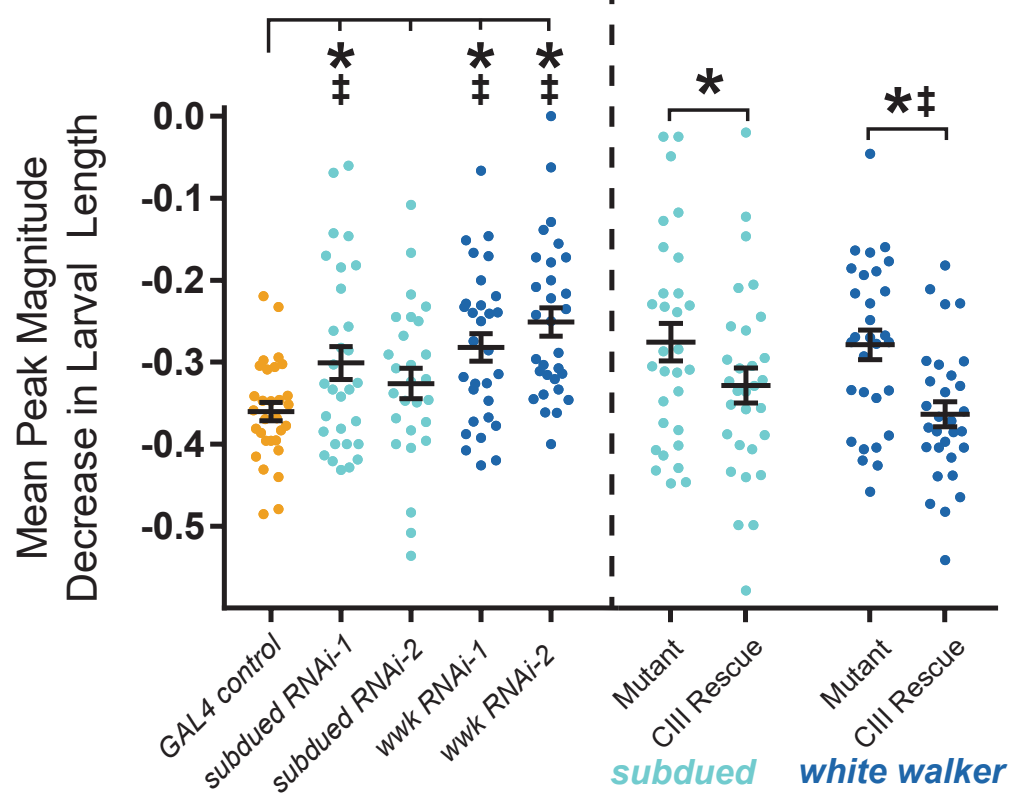

\section{Anoctamins are required for cold-evoked CIII activity}

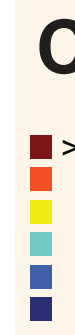

C
$->5$
4
3
2
1
0
0
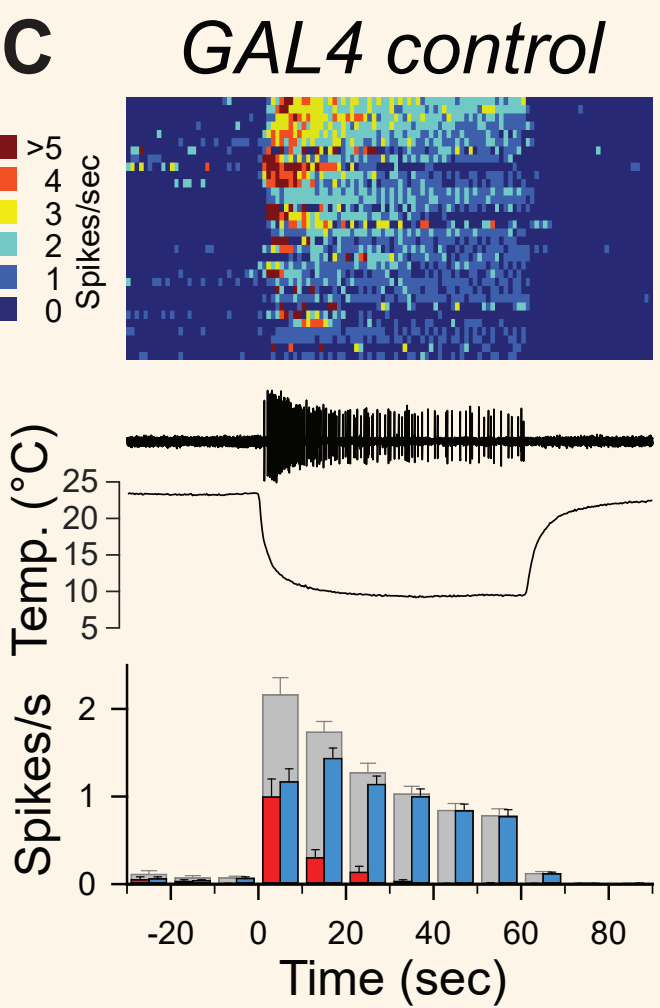

F

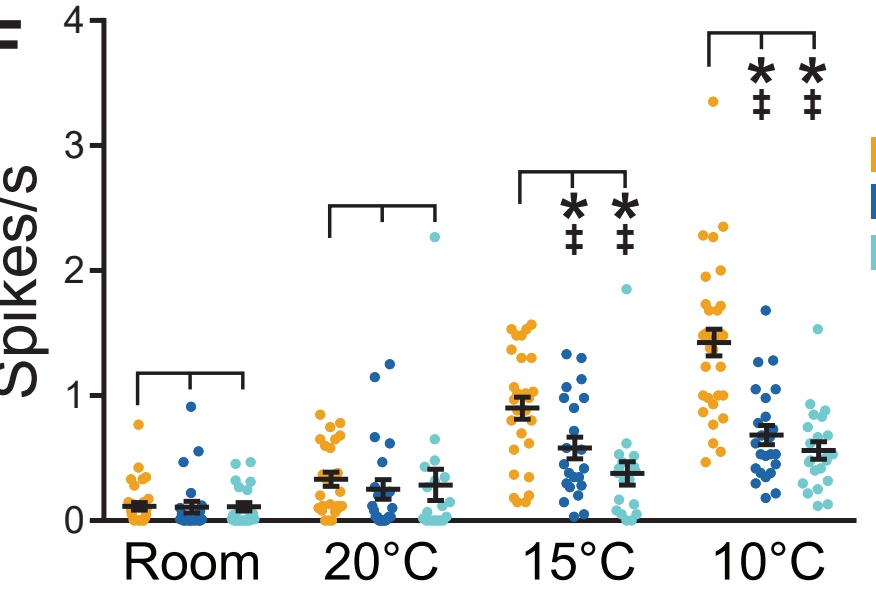

D $w w k R N A i$
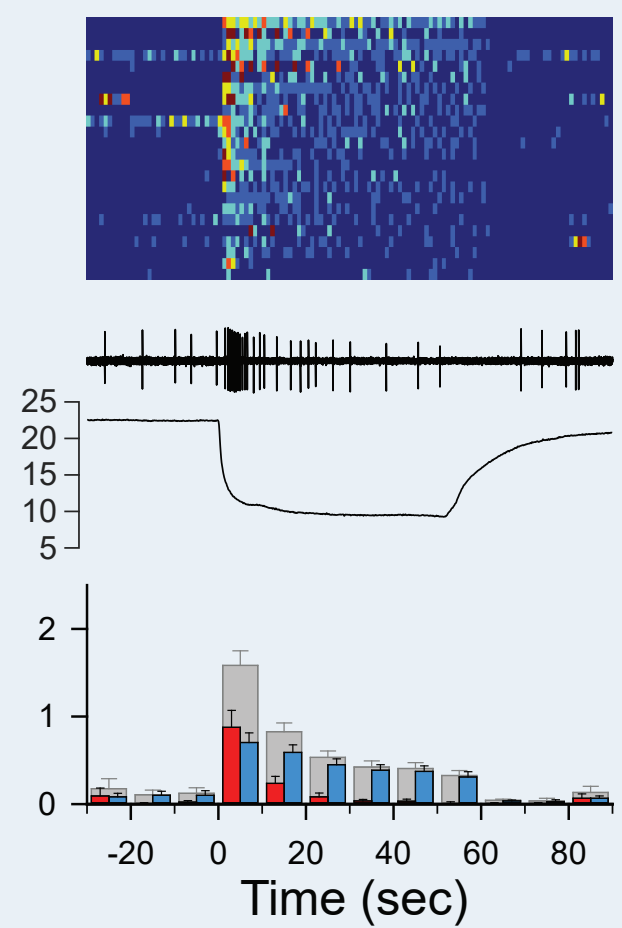

GAL4 control

white walker RNAi

subdued RNAi
E subdued RNAi
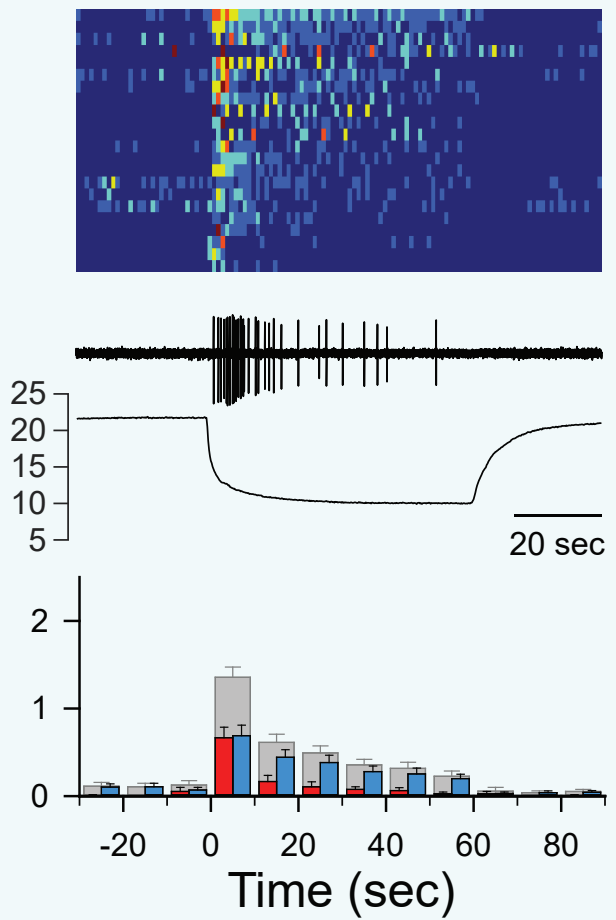

Temp.

All spikes

$\square$ Bursting

Tonic 


\section{Anoctamins are not required for gentle touch mechanosensation}

A

\section{Summed Touch Scores}

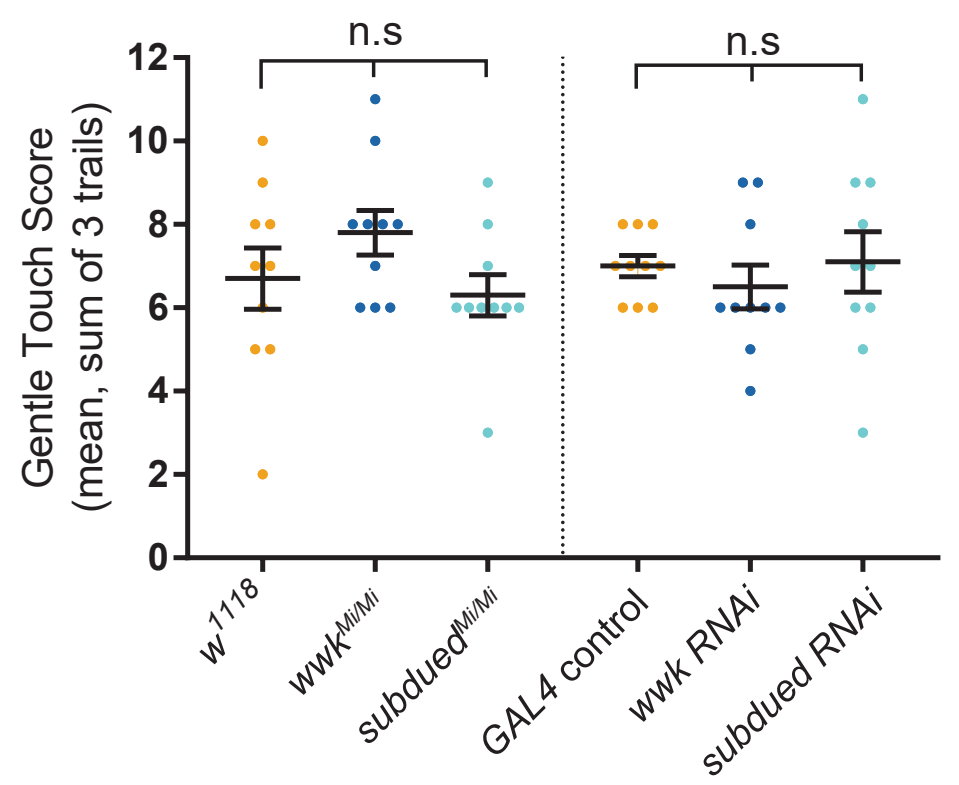

B Individual Trials

mutant

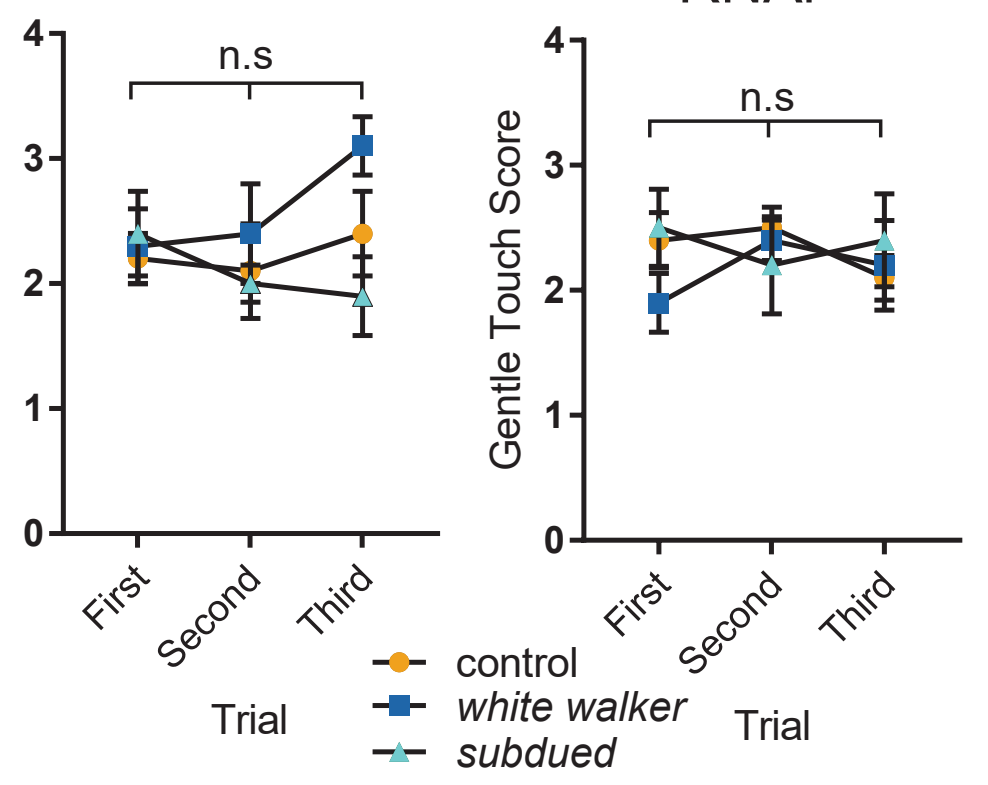

\section{Anoctamins are not required for CIII dendritogenesis}

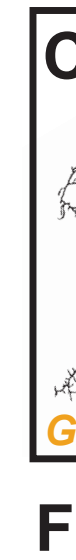

\begin{tabular}{l}
$\mathbf{C}$ \\
$\mathbf{F}$ \\
\hline
\end{tabular}
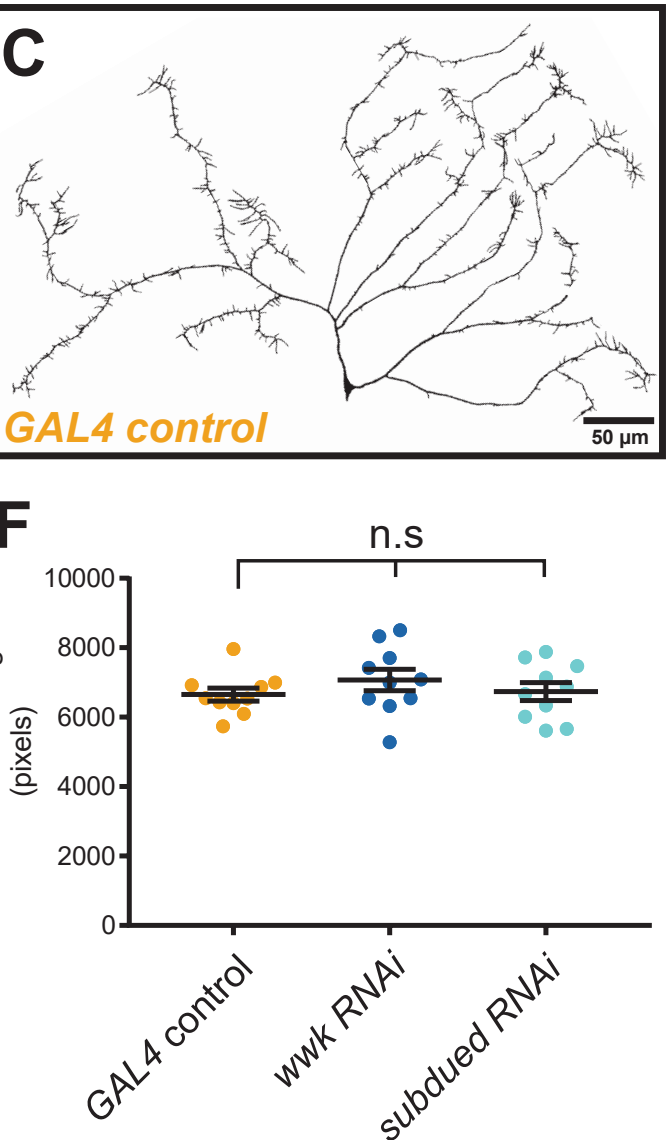
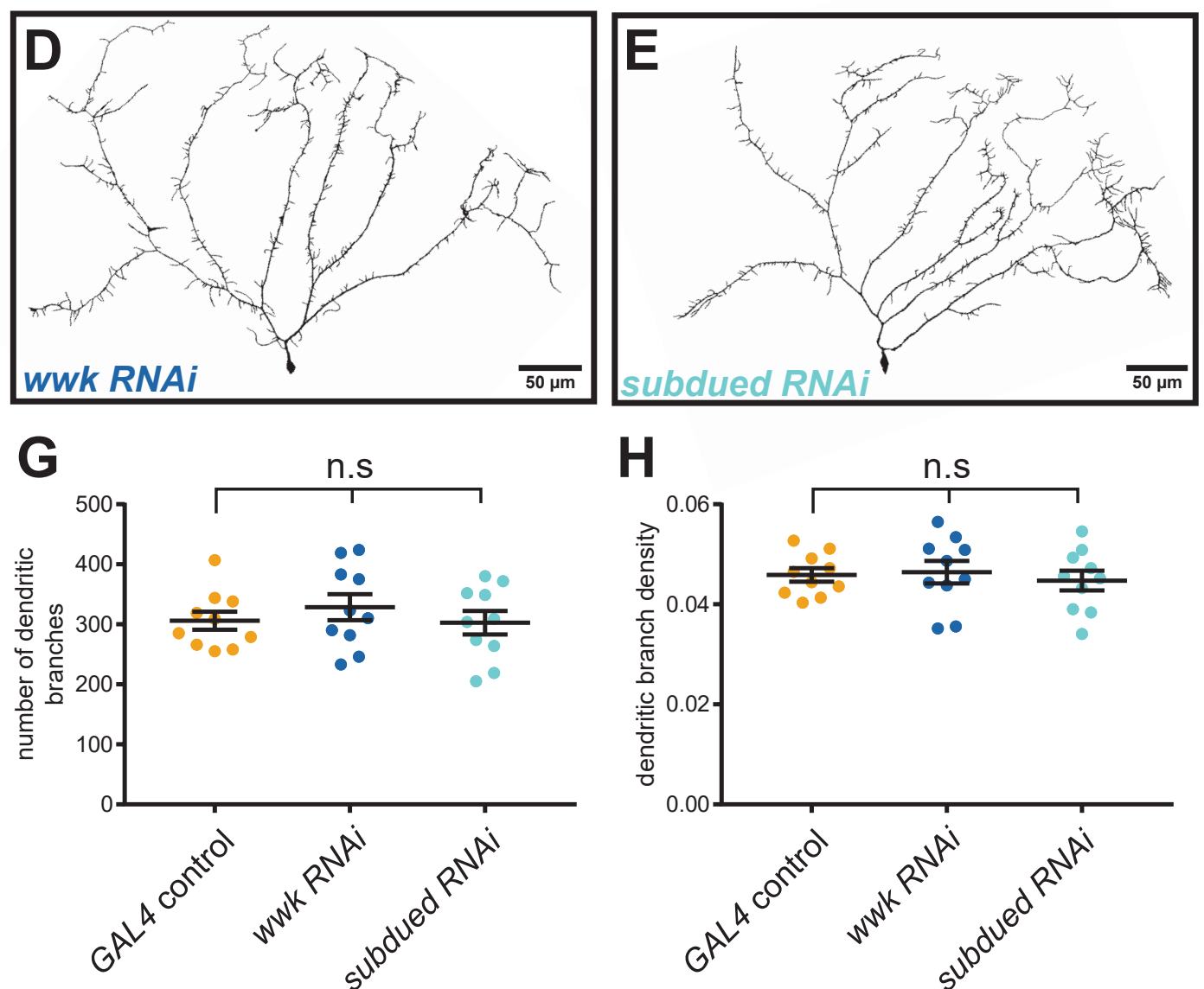

$\mathbf{H}$

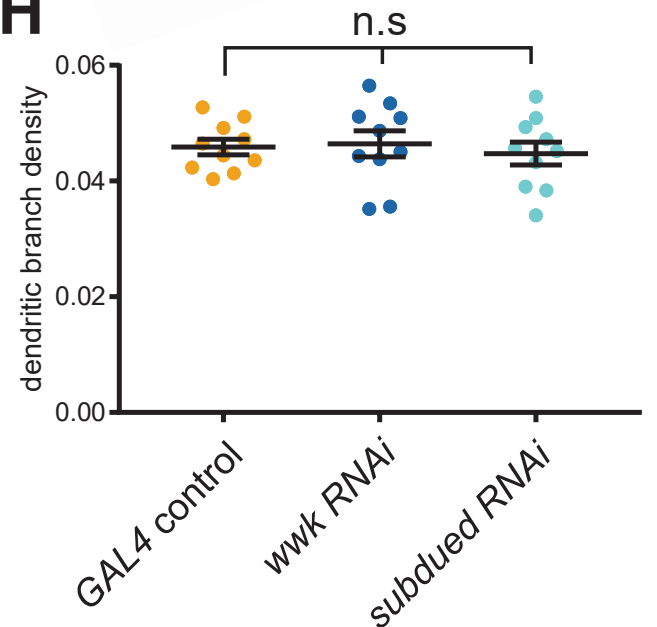




\section{Anoctamin/TMEM16 Channels - 5 metazoan clades}

A

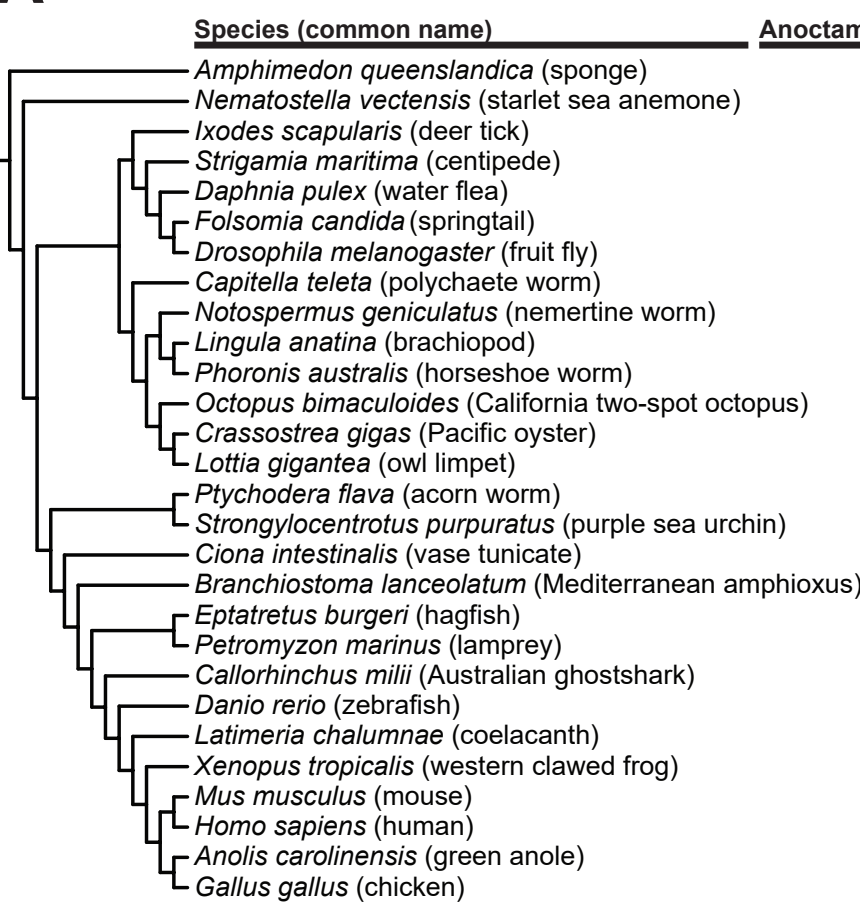

B

\section{white walker}

(CG15270)

2
9
3
4
4
5
5
6
10
3
4
8
7
6
1
6
4
4
7
10
27
15
13
26
10
8
10
26

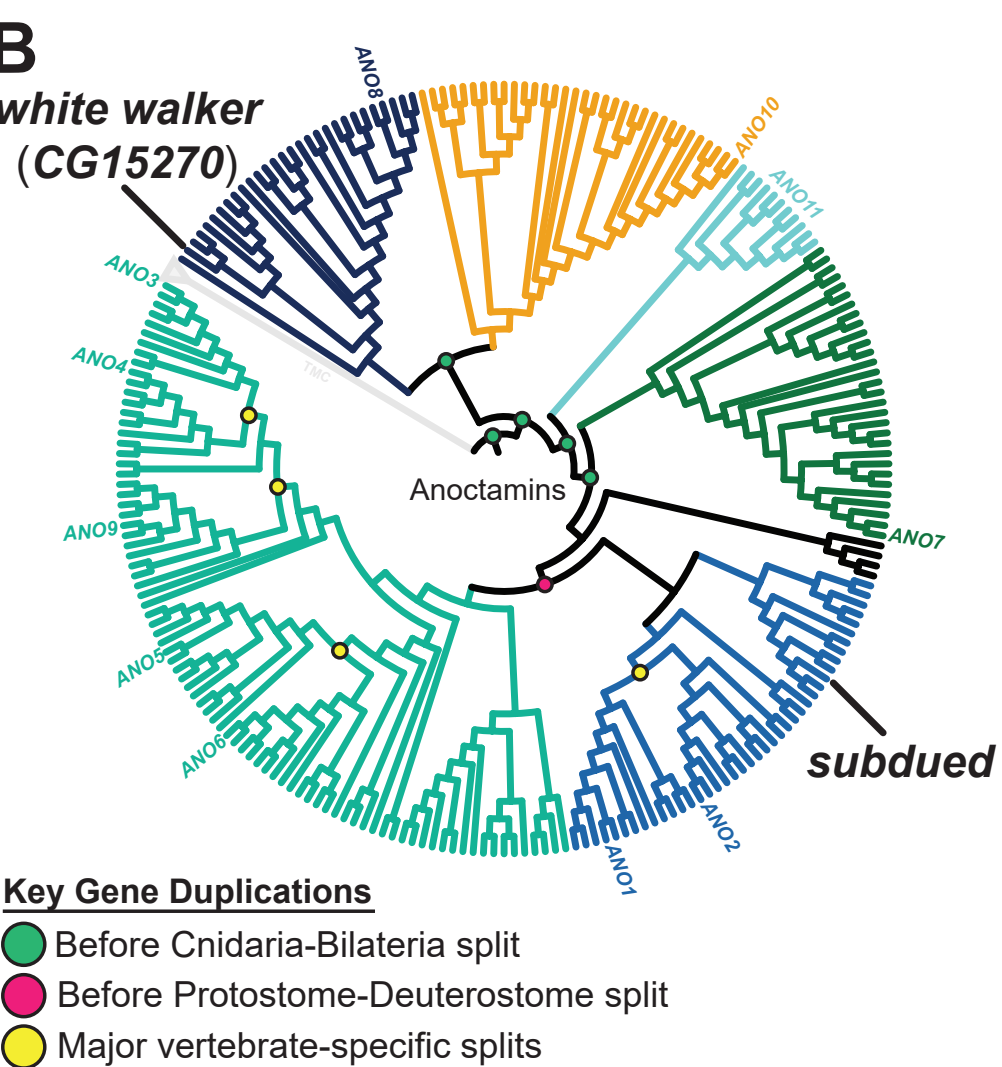


Figure 5

\section{Knockdown of ncc69 reduces CT}

A

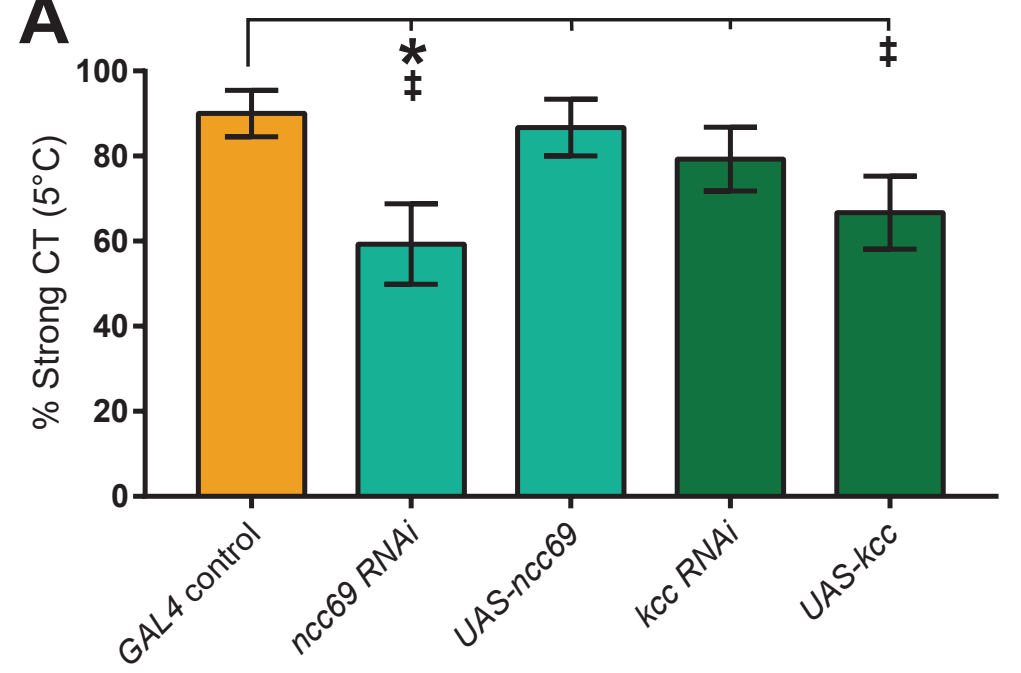

B

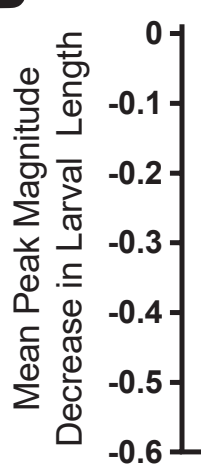

$\stackrel{\star}{\star}$

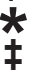

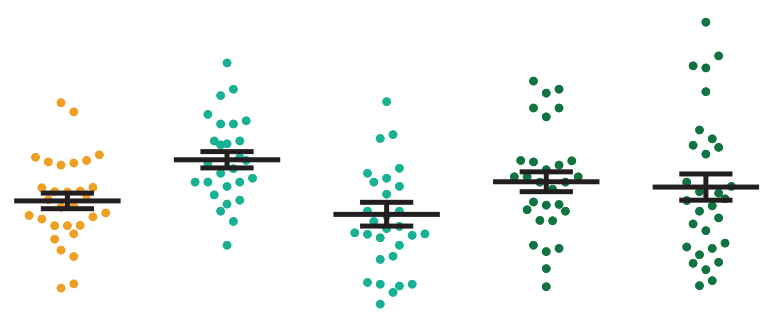

\section{Activation of $\mathrm{Cl}^{-}$channel Aurora activates $\mathrm{CIII}$ neurons}

C Blue light OFF

Blue light ON
D - GAL4 Control

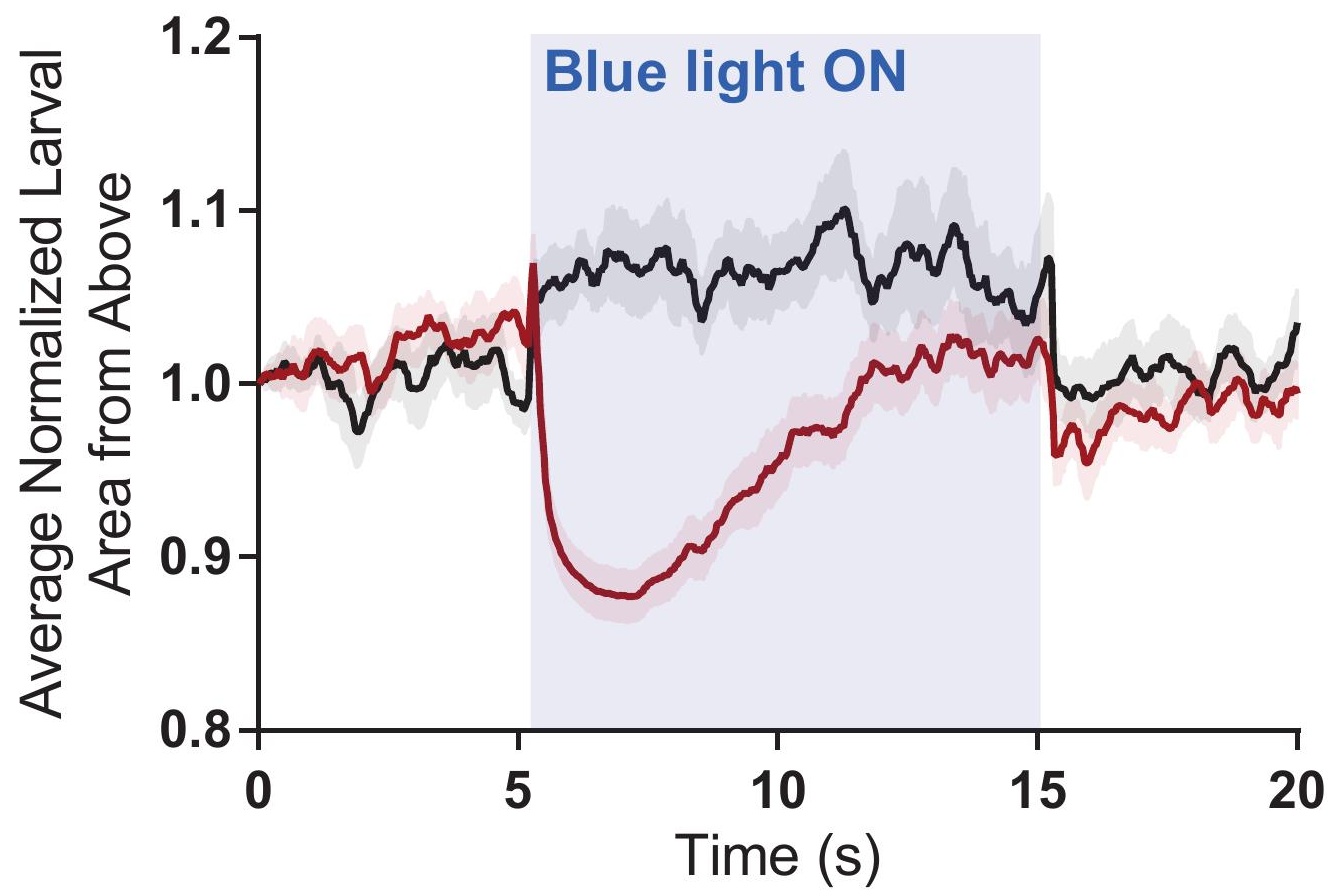


Figure 6

\section{Low extracellular $\mathrm{Cl}^{-}$sensitizes $\mathrm{CIII}$ neurons}

A
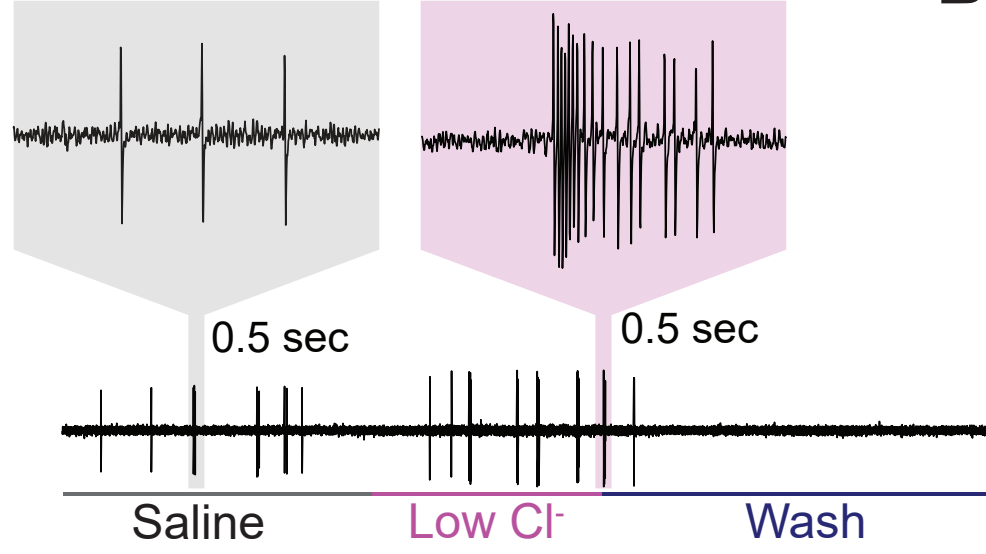

25

20

$\left({ }^{\circ} \mathrm{C}\right)$
B

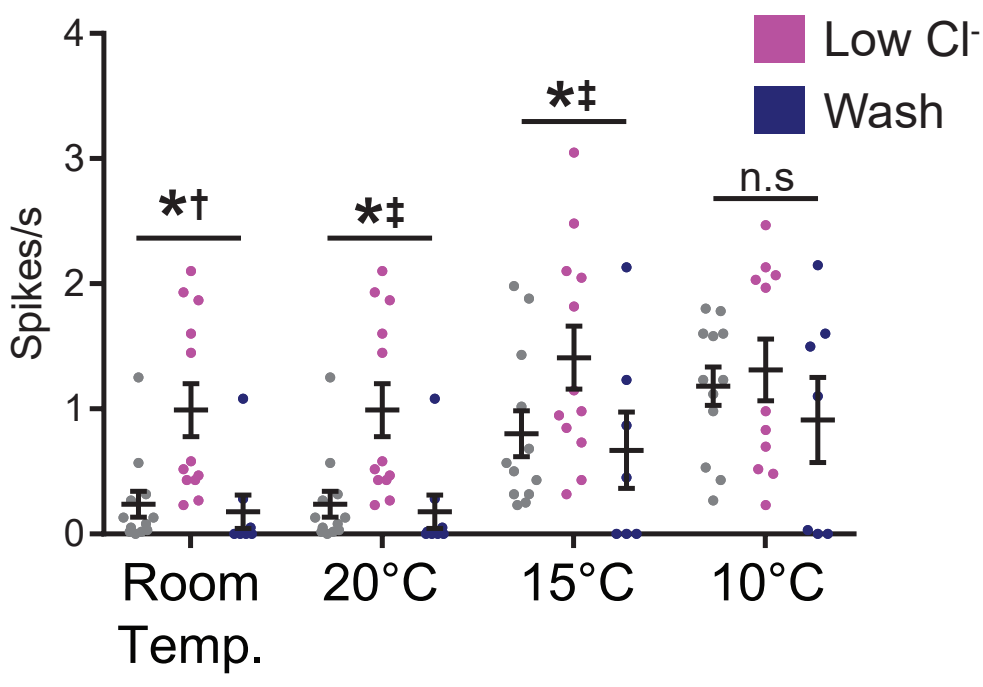




\section{A GAL4 control}
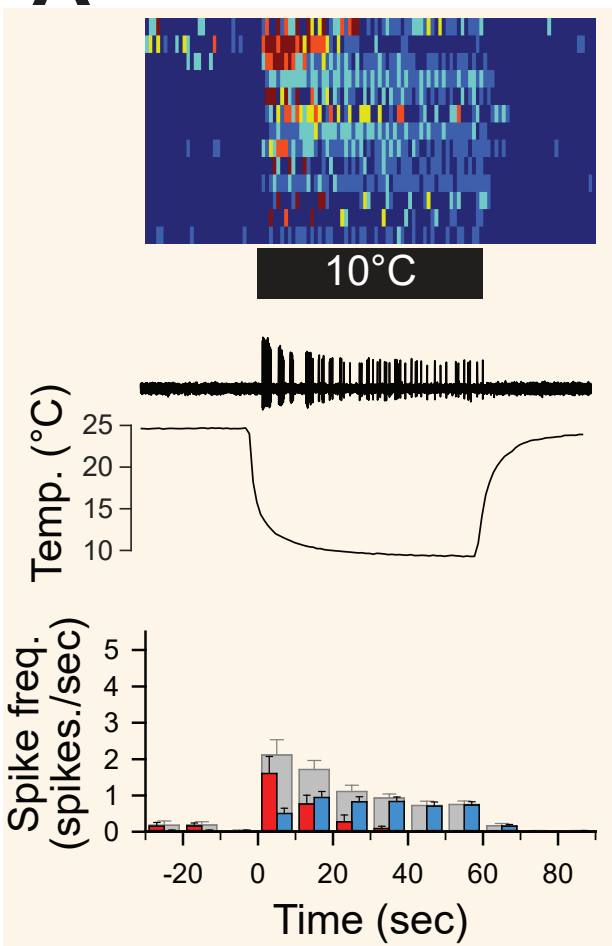

B ncc69 RNAi
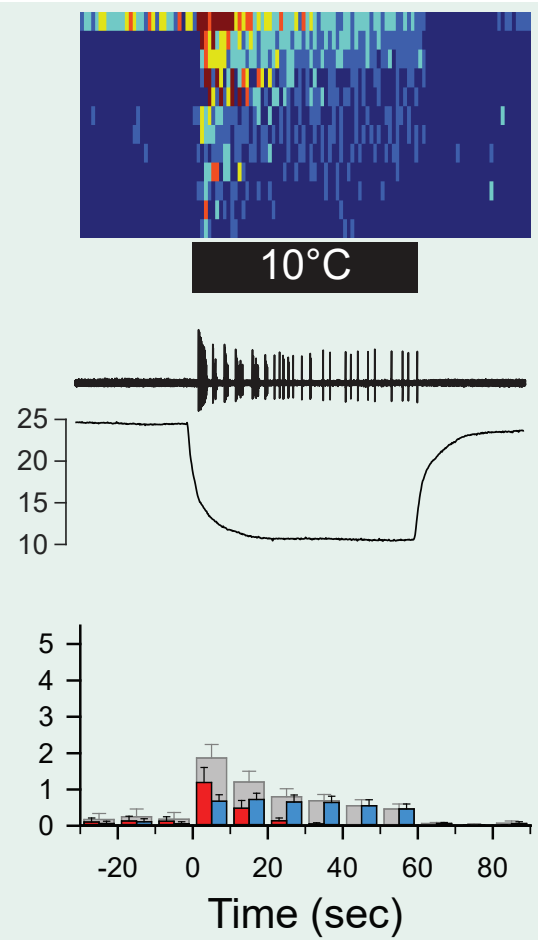

C UAS-ncc69
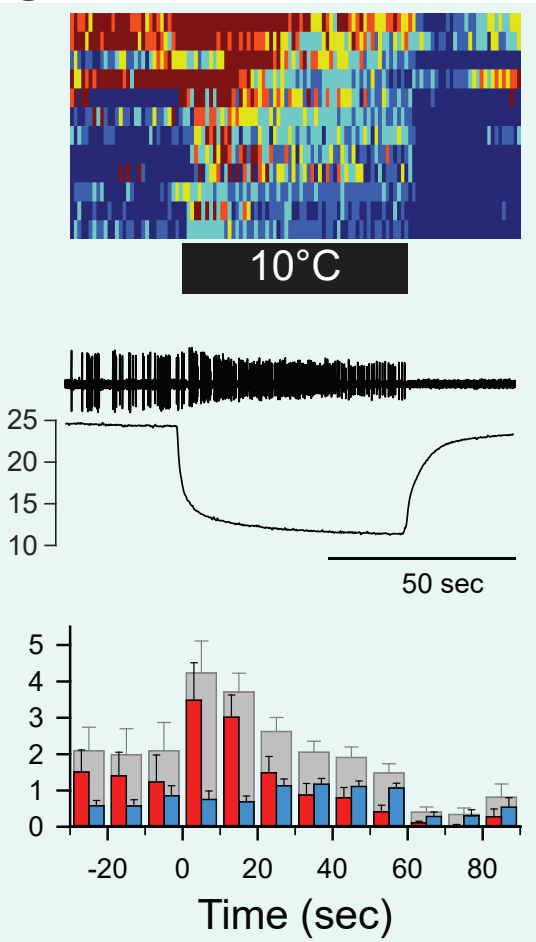

$>5$
4 d
3 के
2 d
1 के
0 के

All spikes Bursting Tonic

D

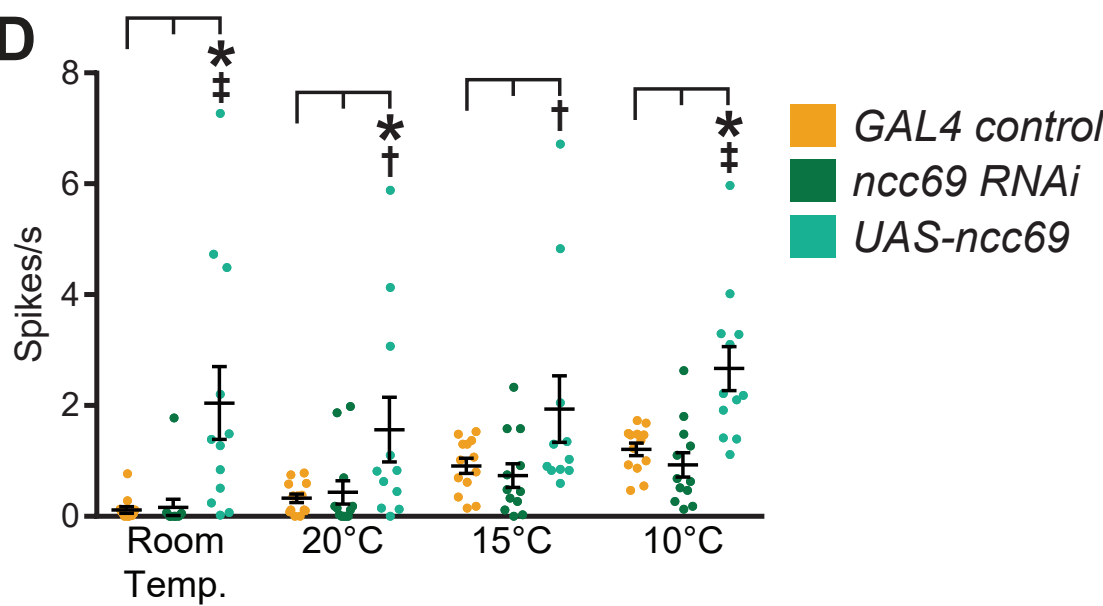

Overexpression of ncc69 sensitizes larvae to less-noxious cold

E

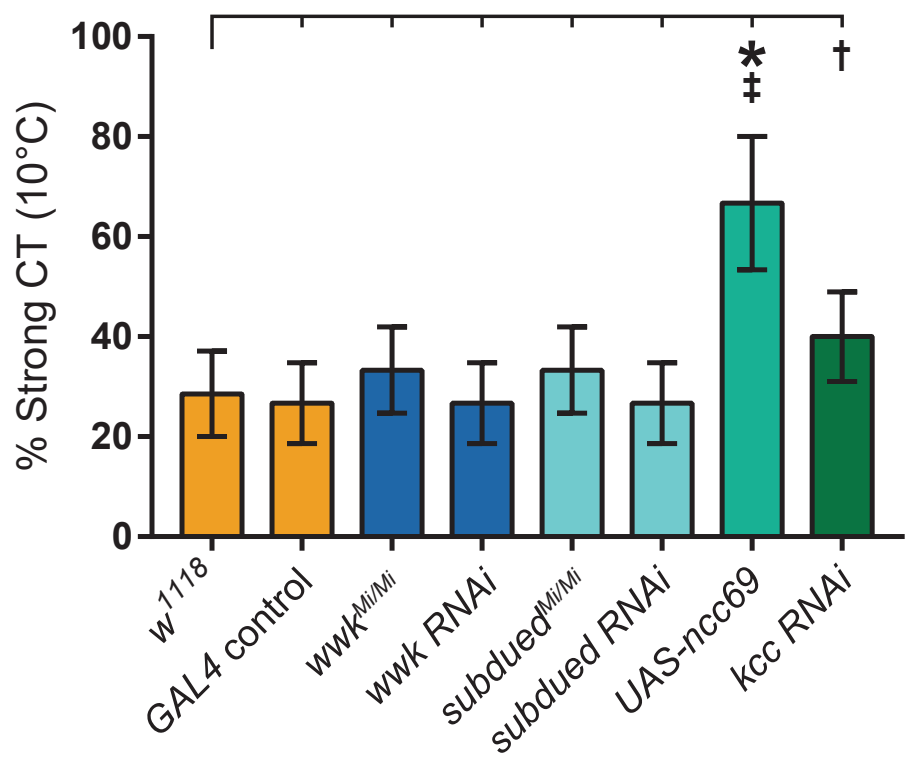

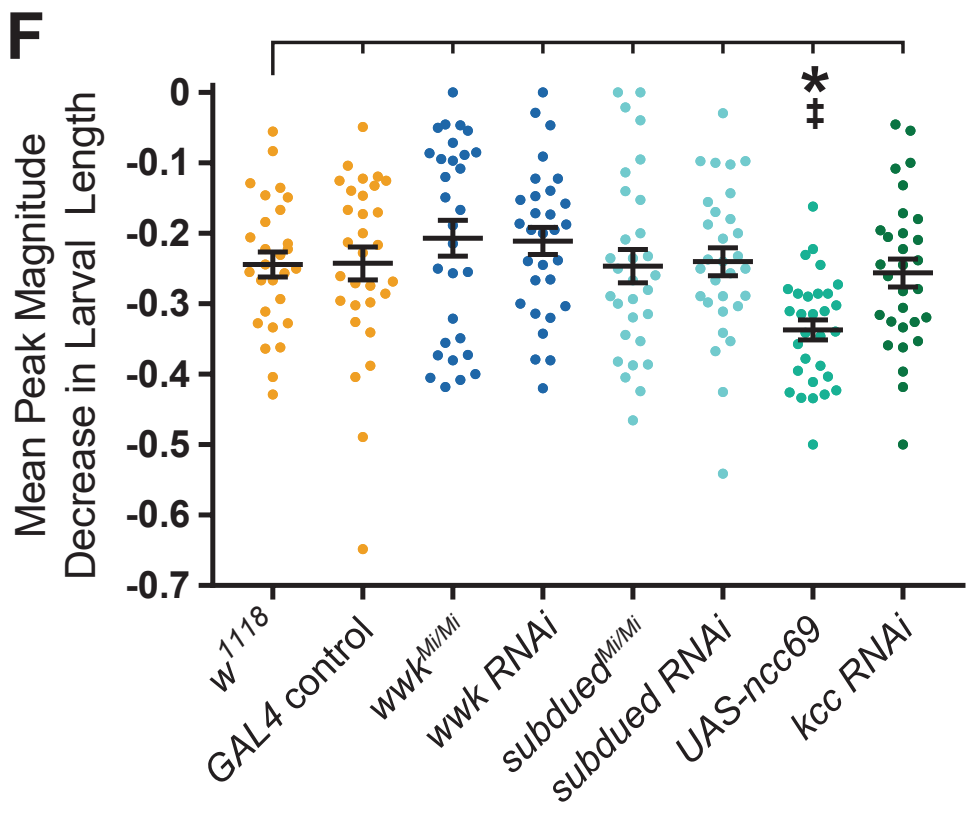


Figure 8

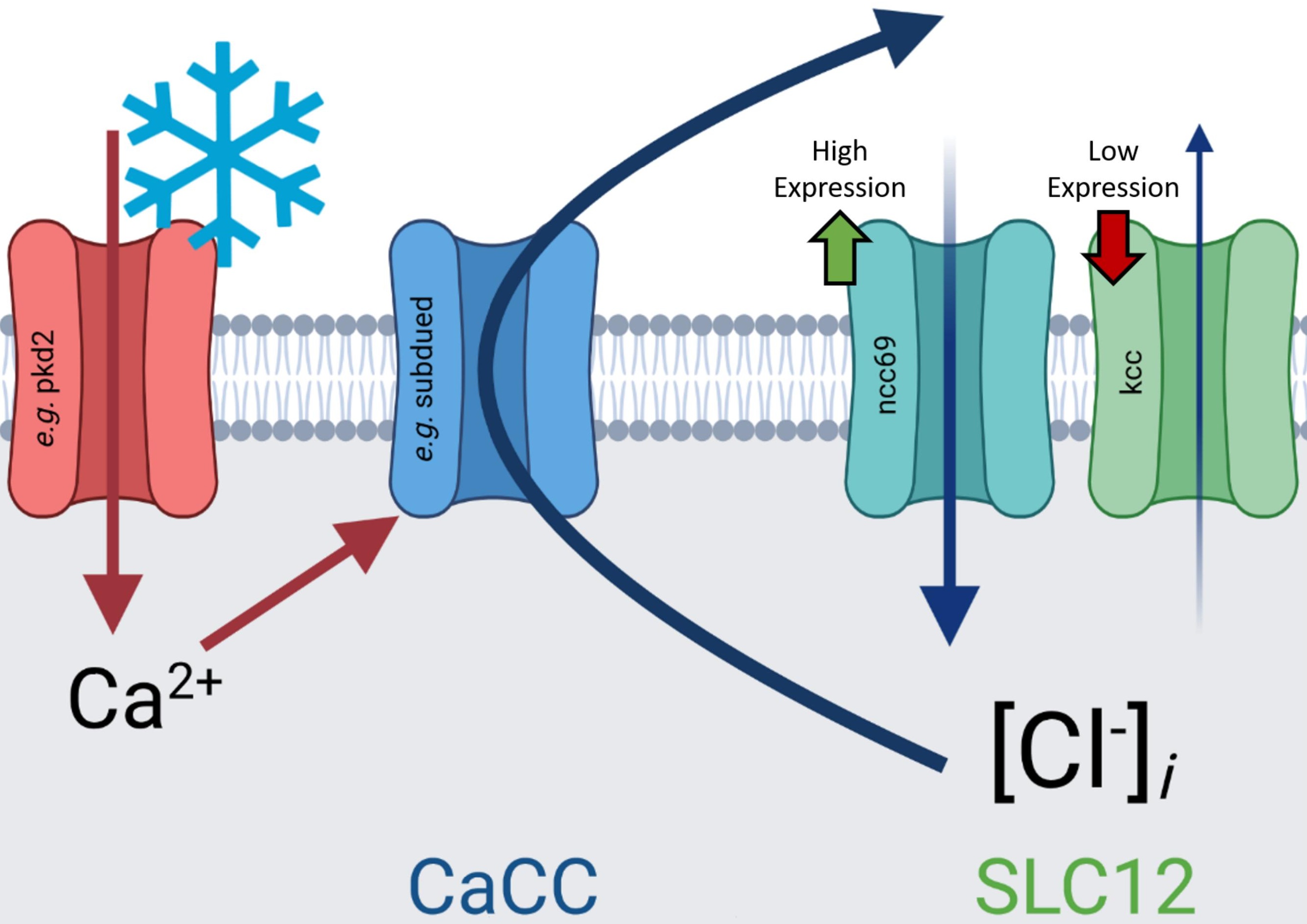

